\title{
A global analysis of SNX27-retromer assembly and cargo specificity reveals a function in glucose and metal ion transport
}

\author{
Florian Steinberg ${ }^{1}$, Matthew Gallon ${ }^{1}$, Mark Winfield ${ }^{2}$, Elaine Thomas ${ }^{1}$, Amanda J. Bell $^{1}$, \\ Kate J Heesom ${ }^{3}$, Jeremy M. Tavaré ${ }^{1}$, and Peter J. Cullen ${ }^{1}$ \\ ${ }^{1}$ The Henry Wellcome Integrate Signalling Laboratories, School of Biochemistry, University of \\ Bristol, Bristol BS8 1TD, UK \\ ${ }^{2}$ School of Biological Sciences, University of Bristol, Bristol BS8 1UG, UK \\ ${ }^{3}$ Proteomics Facility, School of Biochemistry, University of Bristol, Bristol BS8 1TD, UK
}

\section{Abstract}

The PDZ domain-containing sorting nexin 27 (SNX27) promotes recycling of internalized transmembrane proteins from endosomes to the plasma membrane by linking PDZ-dependent cargo recognition to retromer-mediated transport. Here, we employed quantitative proteomics of the SNX27 interactome alongside quantification of the surface proteome of SNX27 and retromersuppressed cells to dissect the assembly of the SNX27 complex and provide an unbiased global view of SNX27-mediated sorting. Over 100 cell surface proteins, many of which interact with SNX27, including the glucose transporter GLUT1, the Menkes disease copper transporter ATP7A, various zinc and amino acid transporters, and numerous signalling receptors require SNX27retromer to prevent lysosomal degradation and maintain surface levels. Furthermore, we establish that direct interaction of the SNX27 PDZ domain with the retromer subunit VPS26 is necessary and sufficient to prevent lysosomal entry of SNX27 cargo. Our data identify the SNX27-retromer as a major endosomal recycling hub required to maintain cellular nutrient homeostasis.

\section{Introduction}

Following internalization, endocytosed transmembrane proteins are delivered to early endosomes. Here, cargo proteins are sorted for degradation in lysosomes or alternatively retrieved and recycled to the plasma membrane or the trans-Golgi network (TGN). The sorting nexins (SNX) of the SNX17-SNX27-SNX31 sub-family are early endosomeassociated proteins that promote the retrieval and recycling of specific cargo ${ }^{1}$. While SNX17 sorts cargoes containing NPxY-NxxY motifs such as LRP $1^{2}$ and $\beta 1$-integrins ${ }^{3,4}$, the PDZ domain-containing SNX27 recycles the $\beta 2$-adrenergic receptor ( $\beta 2 \mathrm{AR})$ via direct binding to a PDZ-ligand located at the C-terminus of the receptor ${ }^{5}$.

\footnotetext{
Author contributions: FS, JMT and PJC designed the project; FS, MG, ET and AJB performed the experiments; MW performed the bioinformatics; KJH performed the proteomics; FS, MG and PJC wrote the manuscript.

Conflict of interest: The authors declare that they have no conflict of interest.
} 
SNX27-mediated retrieval and recycling of the $\beta 2 \mathrm{AR}$ has been proposed to depend on the SNX-BAR-retromer through an indirect interaction with the retromer-associated WASH complex ${ }^{6}$. In mammals, the SNX-BAR-retromer comprises a stable VPS26:VPS29:VPS35 trimer that is loosely associated with heterodimers composed of SNX1-SNX2 and SNX5SNX6 ${ }^{7-11}$. The best-characterised role of this complex is in the retrieval and recycling of cargo from endosomes back to the TGN ${ }^{7-11}$. The proposed link with SNX27 therefore suggests an additional role for SNX-BAR-retromer in direct endosome-to-plasma membrane recycling ${ }^{6}$. Here, we have employed quantitative proteomic analysis of the SNX27 interactome combined with quantification of the surface proteome of SNX27 and VPS35deficient cells to obtain a global view of SNX27-retromer assembly and cargo specificity. This has identified SNX27 as a core component of a multi-protein complex defined by the independent association with the Wiskott-Aldrich syndrome protein and SCAR homologue (WASH) complex and retromer SNX-BARs, and a direct PDZ domain-mediated interaction with the VPS26 component of the VPS26:VPS29:VPS35 trimer. Moreover, our global approach has established SNX27-retromer as a major retrieval and recycling hub for a variety of transmembrane proteins, many of which play crucial roles during organism growth and cellular homeostasis.

\section{Results}

\section{Proteomic analysis of SNX27 and retromer cargo specificity}

To globally identify SNX27 and retromer-dependent cargo, we developed a two-tiered proteomics approach based on stable isotope labeling with amino acids in culture (SILAC): First, we employed high resolution interactome analysis of GFP-trap precipitated GFPSNX27 vs. GFP to identify new SNX27 interactors. Secondly, we comparatively quantified the biotinylated surface proteome of control, SNX27 and retromer (VPS35) suppressed cells through triple SILAC analysis to uncover cargo that depend on these proteins for endosomal recycling and surface abundance. To obtain the interactome, GFP-SNX27 and GFP were lentivirally expressed in SILAC labelled human RPE1 and HeLa cells and precipitated with the highly efficient GFP-trap method ${ }^{12}$. Besides robustly detecting the known interaction partners DGKZ ${ }^{13}, \beta$-Pix, GIT1 and GIT2 $2^{14}$ (Table S1), our screens identified 77 integral membrane proteins that were at least 3-fold enriched over control (Table S2). Many of these SNX27 interactors harbour a class I PDZ ligand at their C-terminus (S-TX $\phi$ ), with an apparent preference for a negative charge (E or $\mathrm{D}$ ) at the -3 position and, as published previously, a lack of a positive charge at the -5 position ${ }^{15}$ (also see text in Table S2). Immunoprecipitation experiments confirmed an interaction with the potential cargoes (Fig. S1A). The surface proteome analysis of SNX27 and retromer deficient cells was then used to functionally validate these hits and also to identify low affinity SNX27 cargo that escaped detection in our interactome screen.

Suppression of SNX27 and VPS35 in the SILAC-labelled cells was highly efficient (Fig. S1B). In 6 independent experiments we used different siRNA oligonucleotides and swapped the SILAC media between groups of three experiments to minimize unspecific and offtarget effects (SILAC workflow is shown in Fig. S1C). Of a total of 4900 quantified proteins, 152 membrane proteins were lost more than 1.4-fold from the surface of VPS35- 
depleted cells compared to control cells (Fig. 1A and Table S3). For SNX27-depleted cells, 122 proteins were at least 1.4-fold decreased. Notably, the majority (81 out of 122) of the proteins lost from the surface of SNX27-suppressed cells were also lost from VPS35deficient cells (Fig. 1A and Table S3), suggesting that most of SNX27-mediated recycling depends on VPS26:VPS29:VPS35 function. In contrast, many of the 152 proteins that were lost from VPS35-depleted cells were not affected by SNX27 suppression (71 out of 152), suggesting that VPS26:VPS29:VPS35 can recycle membrane proteins independently of SNX27. Importantly, 37 out of the 81 proteins that were affected by SNX27 and VPS35 depletion were also enriched in the SNX27 interactome (Fig. 1A, Table S2), making them high confidence cargo. Among the SNX27 interactors that were lost from the surface of SNX27 and VPS35-deficient cells were the glucose transporter GLUT1, the monocarboxylate transporter MCT1, the GPCR-like receptor CD97, the metalloreductase STEAP3, the membrane scaffold KIDINS220, the Menkes disease-linked copper transporter ATP7A, the zinc transporter ZnT1 and a plethora of other transporters and signalling receptors. Among the potential low affinity SNX27 cargo not enriched in the interactome were the TrailR1 death receptor, PDGFR $\beta$, the thrombin receptor PAR1, the TGF $\beta$-receptor 1, two ZIP family zinc transporters (ZIP6, ZIP10), members of the LRP family, the phosphatase PTPRJ and the Alzheimer precursor protein APP. We also detected a loss of the known cargo $32 \mathrm{AR}$ under both SNX27 and VPS35-suppressed conditions, but, probably owing to low expression levels in HeLa and RPE1 cells, failed to detect it in our interactome (Fig. 1A, Table S3). It should be noted that a small number of proteins was also more abundant at the surface of SNX27 and VPS35 depleted cells (Fig. S1D and Table S4).

Fluorescence-based quantitative western blotting of surface fractions of SNX27 and VPS35depleted cells confirmed every hit we validated (Fig. 1B and Table S3). Membrane proteins like the EGFR and N-cadherin, which were not changed in the SILAC data, were also not affected in western blot experiments (Fig. 1B). We also confirmed that CSPG4-NG2, one of the proteins specifically reduced upon VPS35 suppression, was indeed only lost from the surface of VPS35-deficient cells (Fig. 1B). As indicated by the SILAC data, ATP7A was lost from the surface of SNX27-depleted cells (Fig. 1A), a phenotype that became more obvious when plasma membrane translocation was induced by high copper ${ }^{16}$ (Fig. 1C). Furthermore, flow cytometric analysis confirmed that the surface receptors CD97, PTPRJ and TrailR1 were lost from the surface of SNX27 and VPS35-deficient cells to an extent that correlated with the SILAC data (Fig. 1D, Table S3).

\section{SNX27 and retromer cargo is lysosomally degraded upon suppression of SNX27 or VPS35}

Immunofluorescent staining of CD97 and TrailR1 in control and SNX27 or VPS35-depleted cells revealed that each protein was not only lost from the cell surface but severely lost at the whole cell level (Fig. 2A). To determine if these proteins were lost due to enhanced lysosomal degradation, we blocked lysosomal proteases and performed multi-hour uptake assays with antibodies against the ectodomains of endogenous CD97 and TrailR1. In control cells, TrailR1 and CD97 antibodies were mostly localized at the plasma membrane, indicating efficient recycling (Fig. 2B and S2A). In SNX27, and more so in VPS35-depleted cells, we observed strong colocalization with LAMP1-positive lysosomes (Fig. 2B and S1C). Similarly, in steady-state immunofluorescent stainings, endogenous GLUT1 was 
localized to the plasma membrane in control cells but almost completely localized to LAMP1, but not EEA1, positive vesicles in SNX27 and VPS35 suppressed cells (Fig. 2C, S2B and S2C). In agreement with lysosomal mis-sorting, degradation kinetics of GLUT1, MCT1 and ATP7A, but not the controls N-cadherin or TfnR, were increased in SNX27 and VPS35-depleted cells (Fig. 2D). Consistently, levels of GLUT1, ATP7A and MCT1 were reduced in whole cell lysates of SNX27 and retromer depleted cells (Fig. S2D). SNX27 and retromer are therefore required for the post-translational stability of a large cohort of cargo proteins.

\section{SNX27 and retromer cargoes require their PDZ ligands to avoid lysosomal degradation}

SNX27 prevents degradation of the $\beta 2 A R$ through binding to the PDZ ligand present in the C-terminal tail of the receptor ${ }^{5,6}$. In agreement with this, the cytoplasmic tails (or last 40 amino acids) of GLUT1, ATP7A, STEAP3, MCT1, SLC30A1 (ZnT1), SLC4A7, KIDINS220 and CD97, all of which were enriched in our interactome (Fig1 A, TableS2 and Fig. 3A), precipitated endogenous SNX27 but not mCherry-SNX27 $\triangle$ PDZ (Fig. 3A). The tails of PDGFR $\beta$, PTPRJ and TrailR1, which were lost from the surface of SNX27-depleted cells but not enriched in the interactome (Fig. 1A, 1B and 1D), did not precipitate SNX27, suggesting either no direct or low affinity binding (Fig. 3A). In agreement with an interaction, antibody uptake assays revealed that HA-tagged GLUT1 and endogenous CD97 co-localized with GFP-SNX27 and endogenous VPS35 on endosomes (Fig. 3B). If binding of SNX27 to the PDZ ligand is required to prevent lysosomal entry, removal of this sequence should mimic SNX27 suppression. Indeed, myc-tagged GLUT1 resided mainly at the plasma membrane while HA-tagged GLUT1 $\triangle \mathrm{PDZ}$ (lacking the last 3 amino acids) localized to LAMP1-positive lysosomes (Fig. 3B). Finally, consistent with GLUT1 being the primary glucose uptake transporter in many cell types ${ }^{17}$, SNX27 and VPS35 suppression led to a more than $60 \%$ decrease of glucose uptake in HeLa cells (Fig. 3D).

\section{Retromer SNX-BARs are dispensable for lysosomal rescue but mediate efficient transport}

As the SNX27 cargo 32 AR can enter SNX1 decorated tubules in a SNX27-dependent manner ${ }^{6}$, we addressed whether suppression of SNX1, SNX2, SNX5 and SNX6 would result in a similar phenotype as depletion of SNX27 or VPS35. Since SNX1 and SNX2 as well as SNX5 and SNX6 are functionally redundant ${ }^{10,11}$, we performed double SNX1SNX2 and SNX5-SNX6 suppression and quantified surface localization and lysosomal missorting of the GLUT1 transporter. Suppression of the SNXs had a mild but significant impact on surface levels of GLUT1, with similar effects being observed for ATP7A and STEAP3(Fig. 4A). Image analysis revealed that GLUT1 localization was shifted from the plasma membrane to intracellular vesicles upon SNX1-SNX2 or SNX5-SNX6 suppression (Fig. 4B). Interestingly, these vesicles were not lysosomes but were positive for the early endosome marker EEA1 (Fig. 4C). Consistent with this, GLUT1 and ATP7A were not subject to increased lysosomal degradation in SNX1-SNX2 or SNX5-SNX6-depleted cells (Fig. 4D). The retromer associated SNX-BARs are therefore not necessary to prevent entry of SNX27-retromer cargo into the lysosomal pathway, but are involved in subsequent transport from an EEA1-positive, SNX27 and retromer decorated compartment. 


\section{SNX27 is a component of the SNX-BAR retromer}

SNX27 has been proposed to link to retromer via an indirect interaction through the retromer associated WASH complex ${ }^{6}$. However, our SILAC interactome data (Table S1) and western blotting experiments suggested that all retromer associated SNX-BAR proteins (SNX1, SNX2, SNX5 and SNX6), the VPS26:VPS29:VPS35 trimer and the WASH complex were highly enriched in the SNX27 interactome (Fig. 5A). Interactions were specific for SNX27 as sub-family members SNX17 or SNX31 did not precipitate any interacting partner (Fig. 5A). The interaction of SNX27 with other sorting nexins was specific for retromer SNX-BARs, as we did not detect SNX4, a SNX-BAR previously linked with endosomal recycling ${ }^{18}$ (Fig. 5A). Furthermore, triple SILAC proteomics and immunoprecipitations on GFP versus GFP-SNX27 versus GFP-SNX27 $\triangle \mathrm{PDZ}$, a deletion mutant lacking the entire PDZ domain (Fig. 5B), revealed that deletion of the PDZ domain completely abolished enrichment of the VPS26:VPS29:VPS35 trimer while binding to the WASH complex and the retromer SNX-BARs was retained (Fig. 5C and Table S5). As these findings stood in contrast to the proposed model for SNX27:SNX-BAR-retromer assembly we further investigated these interactions.

\section{The SNX27 FERM-like domain associates independently with WASH components and the retromer SNX-BARs}

To map the above interactions, SNX27 was split into GFP-tagged sub-domains defined by the PDZ domain, the phosphoinositide-binding PX-domain and the FERM-like domain ${ }^{19}$, which was also further split into its subdomains F1, F2 and F $3{ }^{19}$. Precipitation of these domains revealed: The PDZ bound the VPS26:VPS29:VPS35 complex, the FERM-like F3 subdomain mediated WASH binding while the entire FERM domain was needed to precipitate the SNX-BAR proteins (Fig. 6A, for an overview also see Fig. 7J). Based on these data, we created a SNX27 $\triangle$ FERM construct and a SNX27-SNX17 chimera, in which the SNX27 FERM-like domain was replaced with the non-WASH complex binding FERM domain of SNX17 (see Fig. 5A). These mutants bound the VPS26:VPS29:VPS35 complex but not the WASH complex or the retromer SNX-BARs (Fig. 6B).

Further experiments revealed that, among sorting nexins, strong binding to the WASH complex was unique to SNX27 (Fig. 6C and D). Unlike the binding to VPS35, which is mediated through the tail region of FAM21 ${ }^{20,21}$, deletion of the FAM21 tail retained SNX27 binding, indicating a distinct mode of binding (Fig. 6E). As VPS35 depletion results in a loss of WASH from endosomes ${ }^{20-22}$, we explored whether SNX27 suppression had a similar effect. While a mild increase in cytosolic WASH1 under VPS35 suppressed conditions was observed, we did not detect a similar phenotype upon SNX27 suppression or a cumulative loss upon co-suppression of SNX27 and VPS35 (Fig. 6F). Endosomal association of WASH1 is therefore partially dependent on VPS35 but not SNX27.

Finally, in agreement with SNX27 binding to the retromer SNX-BARs, SNX1 and to a lesser extent SNX2, but not SNX5 and SNX6, precipitated endogenous SNX27 (Fig. 7A). GFP-tagged SNX1 co-expressed with its physiological dimerization partner SNX6 improved the efficiency of this immunoprecipitation (Fig. 7B). Importantly, in recombinant binding assays with bacterially expressed proteins the FERM-like domain directly bound SNX1 
(Fig. 7C). Overall, the SNX27 FERM-like domain mediates independent association with the WASH complex and direct binding to retromer SNX-BARs.

\section{An evolutionary conserved loop in the SNX27 PDZ domain mediates binding to VPS26}

Immunoprecipitation from HEK293 cells as well as direct recombinant binding assays revealed that the SNX27 PDZ domain directly bound to the retromer subunit VPS26 (Fig. 7D and E). To investigate the potential for an internal PDZ ligand we split VPS26 into its two lobes ${ }^{23}$. While the C-terminal lobe retained VPS35 binding, neither of the lobes precipitated SNX27, suggesting that intact VPS26 and not an internal PDZ ligand was necessary for SNX27 binding (Fig. 7F). To further rule out a classic PDZ interaction, we precipitated endogenous SNX27 with a GFP-tagged PDZ ligand that conformed to the ideal binding preference of SNX27 (YRDTEL, ${ }^{15}$ ). This ligand efficiently precipitated endogenous SNX27 and VPS35 (Fig. 7G). Furthermore, overexpression of mCherry-SNX27 strongly increased the amount of VPS35 and VPS26 precipitating with the PDZ ligand, even though the PDZ domain-binding pocket was blocked as verified by an inability to precipitate the endogenous PDZ ligand-containing SNX27 interactors MRP4 ${ }^{24}$ and KIDINS220 (Fig. 7G). Therefore, VPS26:VPS29:VPS35 binding to the SNX27 PDZ domain can occur simultaneously with cargo engagement through a PDZ ligand. To investigate if VPS26 also binds to other PDZ domains, we performed SILAC-based proteomics on GFP-tagged VPS26 vs. GFP. In addition to confirming SNX27 as one of the major VPS26 interaction partners, this screen detected most interactors that have been reported for retromer (Table S6): VPS29 and VPS35, all components of the WASH complex, the putative Rab7-GAP $\mathrm{TBC}_{1 \mathrm{D} 5}{ }^{25}$ as well as the retromer interactor FKBP15 ${ }^{22}$. The only other enriched PDZ domain-containing protein was the tight junction protein $\mathrm{ZO}-2$, which precipitated indirectly via SNX27 since it was a major interactor in the SNX27 interactome (Table S1). To identify the SNX27 PDZ:VPS26-binding site we compared the PDZ domain of SNX27 with those of NHERF1 and NHERF2, which did not bind VPS26 (Fig. 7H). Alignment of the SNX27 PDZ domain with the NHERF proteins revealed a stretch of 13 amino acids (Leu ${ }^{67}$ to His ${ }^{80}$ ) that clearly differed from all four NHERF PDZ domains (Fig. S3A). This evolutionary conserved stretch (Fig. S3B), constitutes an exposed loop in the SNX27 PDZ domain crystal structure ${ }^{15}$ that is not present in the NHERF1 (PDB:2OZF) and NHERF2 PDZ domains (PDB:2HEA 26; Fig. S3C). Excision of residues 67-77 from full length GFP-SNX27 fully abrogated VPS26-binding but left PDZ ligand-binding intact (Fig. 7I). Overall, SNX27 is a core component of a multi-protein complex defined by the independent FERM-like domainmediated association with the WASH complex and retromer SNX-BARs, and a direct PDZ domain-mediated interaction with the VPS26:VPS29:VPS35 trimer (Fig. 7J). We term this complex the SNX27-retromer.

\section{Functional analysis of the SNX27 interactions in GLUT1 lysosomal rescue}

To integrate our data on SNX27-retromer assembly with functional implications for lysosomal retrieval, we performed SNX27 suppression and rescue experiments using GLUT1 as a model cargo. Specifically, we tested if the WASH complex binding deficient SNX27 $\triangle$ FERM and SNX27-SNX17 chimera, the VPS26 binding deficient SNX27 $467-77$, wild-type SNX27 as well as only the PDZ domain could rescue lysosomal mis-sorting of GLUT1 in HeLa cells deficient of endogenous SNX27. We also tested a chimera in which 
the PDZ domain of SNX27 was swapped with the well-characterised GLUT1-binding PDZ domain of GIPC1 27,28 (Fig. 8A shows an overview of these SNX27 mutants). As expected, this chimeric protein precipitated GLUT1 via its PDZ domain and the WASH complex via the SNX27 FERM-like domain but failed to precipitate VPS26:VPS29:VPS35 (Fig. 8A). Importantly, in immunoprecipitation experiments all constructs retained GLUT1 binding but varied in their ability to associate with VPS26:VPS29:VPS35 and the WASH complex (Fig. 8A). To test for rescue, SNX27 was depleted from HeLa cells and the siRNA-resistant GFPtagged SNX27 mutants were transiently re-expressed with lysosomal mis-sorting of endogenous GLUT1 being assessed by a visual scoring assay in at least 150 cells for each condition. All the constructs that bound VPS26 (SNX27WT, SNX27 4 FERM, SNX27SNX17-chimera, isolated PDZ domain) efficiently rescued GLUT1 mis-sorting while all SNX27 variants that lost VPS26 binding (SNX27 $\triangle$ PDZ, SNX27 $667-77$, GIPC-SNX27) did not rescue. A loss of WASH-complex binding (SNX27 $\triangle$ FERM, SNX27-SNX17-chimera, PDZ domain only) had no effect on lysosomal retrieval of GLUT1 (Fig 8B and 8C). Quantitative analysis of GLUT1 and LAMP1 colocalization confirmed the results from the scoring assay (Fig. 8D). As a further control, the SNX27 constructs did not rescue lysosomal accumulation of GLUT1 in a VPS35-depleted background (Figure S4). We conclude that binding of SNX27 to the WASH complex is neither necessary nor sufficient for its role in lysosomal retrieval. Instead, binding of VPS26 to the PDZ ligand-engaged PDZ domain is the crucial step in cargo retrieval mediated by the SNX27-retromer.

\section{Discussion}

SNX27 displays two independent associations with the SNX-BAR-retromer: the FERM-like domain directly interacts with SNX1 (and likely SNX2), while the PDZ domain directly binds to VPS26. Our results suggest that the function of the SNX27-retromer could be subdivided into two separate processes: retrieval from lysosomal degradation followed by tubular-based plasma membrane recycling. The interaction of the PDZ domain with the trimeric VPS26:VPS29:VPS35 complex is necessary and sufficient to prevent entry of SNX27-dependent cargo into the lysosomal pathway. Once retrieved, cargo enters SNXBAR decorated tubules for transport from early endosomes (Fig. 8E). Indeed, the $\beta 2 \mathrm{AR}$ enrichment in SNX1-decorated tubules is SNX27-dependent ${ }^{6}$. We detected a mild loss of the SNX27-retromer cargoes GLUT1, ATP7A and STEAP3 from the surface of SNX-BARdepleted cells and observed changes in GLUT1 localization that indicated a trafficking delay but not lysosomal mis-sorting. Here, the interaction of the FERM-like domain with SNX1SNX2 could facilitate tubule entry after retrieval through the SNX27-retromer. However, since SNX-BAR suppression had only minor effects on surface localization, other carriers probably substitute for transport once SNX27 and the VPS26:VPS29:VPS35 complex have prevented entry into the lysosomal pathway.

Our study does not address how SNX27 and the VPS26:VPS29:VPS35 complex prevent cargo from entering the lysosomal pathway. One possible explanation is that VPS26:VPS29:VPS35 and the WASH complex define actin-enriched subdomains that spatially separate incoming cargo from ESCRT-decorated subdomains on the endosomal limiting membrane. Actin-stabilized microdomains have been shown to promote recycling of the $32 \mathrm{AR}^{29}$, and these may be similar to a VPS26:VPS29:VPS35-WASH decorated 
subdomain. According to such a model, endocytosed and PDZ ligand-containing cargo would be captured by VPS26:VPS29:VPS35 bound SNX27, thereby enriching the cargo in a rescue-recycling subdomain of an early endosome. Since the interaction of SNX27 with the WASH complex is not needed for lysosomal retrieval of GLUT1, the functional role of this interaction remains unclear. We have refrained from further analyzing the role of the WASH complex in SNX27-retromer mediated sorting because loss of WASH1 results in collapsed endosomal and lysosomal networks, with associated pleiotropic effects on endosomal sorting ${ }^{30}$.

Besides gaining insight into the mechanism of SNX27-retromer function, we have identified a surprisingly large number of VPS26:VPS29:VPS35-dependent cargo, of which a subset are SNX27 dependent. The clear segregation between these groups argues for the proposed function of SNX27 as a cargo adaptor ${ }^{6}$ and against a role of SNX27 in general VPS26:VPS29:VPS35 function. Within the newly identified proteins, the SNX27-dependent cargo can be considered as high confidence hits: many harbour PDZ ligands, are enriched in the SNX27 interactome in a PDZ-dependent manner and precipitate endogenous SNX27 with their isolated C-terminal tails. The PDZ ligand of GLUT1 is crucial for maximum surface localization and cellular stability ${ }^{28}$, as we have confirmed in the present study. Hence, it is likely that the PDZ ligands of many, if not all of the SNX27 interactors confer prolonged protein half-lives through SNX27-retromer mediated retrieval and subsequent recycling. Importantly, SNX27 also harbours a functional FERM-like domain, which, like its family members SNX17 and SNX31, can engage NPxY-NxxY-based sorting motifs 19,31. Several members of the NPxY-NxxY-containing Low density lipoprotein receptor like (LRP) family, LRP5, LRP8 and LRP10 as well as the very low-density lipoprotein receptor (VLDLR) and APP are significantly lost from the surface of SNX27-depleted cells, suggesting that SNX27 may also promote retrieval and recycling of cargo via binding of its FERM-like domain to NPxY-NxxY motifs. Interestingly, we also detected a small number of proteins with increased surface abundance in SNX27 and VPS35 depleted cells. This may reflect a change in the balance of sorting processes in retromer deficient cells as had been previously observed for CI-MPR ${ }^{9}$. However, the number of proteins and the fold-increase over control were low compared to the down-regulated proteins. Moreover, there was limited overlap between the affected proteins in SNX27 and VPS35 depleted cells as well as limited overlap with the SNX27 interactome.

We have also identified a range of potential new cargo that depend on the VPS26:VPS29:VPS35 complex for their surface abundance, among them integrin a5 and a3, several plexins, members of the TNFR family and many transporters. One of our hits, the Transferrin receptor (TfnR), has been shown to enter retromer tubules and a slight trafficking delay under VPS35 depleted conditions was reported ${ }^{6}$. Consistent with this, we detected a moderate (1.5-fold, $\mathrm{p}=0.0002$ ) loss from the surface of VPS35 but not SNX27 suppressed cells. However, we did not detect any increased degradation of TfnR in VPS35 or SNX27 deficient cells. Similarly, integrin a5, which depends on the WASH complex for efficient trafficking ${ }^{32}$, was lost from the surface of VPS35 deficient cells (2-fold, $\mathrm{p}=0.0007$ ) but not degraded under either WASH or VPS35 suppressed conditions ${ }^{3,32}$. While it remains to be determined if only SNX27 cargo or also a subset of the VPS35 dependent cargo is degraded in the absence of retromer function, our proteomic dataset clearly shows that the 
trimeric VPS complex can function independently of SNX27 in the recycling of membrane proteins back to the cell surface. Unlike SNX27 cargo, which is sorted directly back to the plasma membrane in a Rab4 dependent manner ${ }^{6}$, VPS35 dependent cargo could conceivably travel via the retrograde pathway to the TGN and from there back to the cell surface. In summary, our data has established SNX27-retromer as a major endosomal hub that is required to prevent degradation of a broad spectrum of cell surface proteins.

\section{Online methods}

\section{Antibodies}

Antibodies used in the study were: mouse monoclonal SNX27 antibody (Clone 1C6, Abcam, AB77799, 1:500), monoclonal SNX1 (Clone51/SNX1, BD 611482, 1:1000), SNX2 (Clone 13/SNX2, BD 611308, 1:1000), SNX6 (Clone SNX6-7g, Sigma, S6324, 1:500), goat polyclonal SNX5 (Abcam 5983, 1:500), rabbit polyclonal SNX17 (gift from Dr. Guojun Bu, Washington University, 1:1000), rabbit polyclonal SNX4 (Sigma HPA005709, 1:500), rabbit polyclonal WASH1 and Fam21 (gift from Dr. Dan Billadeau, Mayo Clinic, Rochester, 1:1000), rabbit polyclonal Strumpellin antibody (Santa Cruz, 87442, 1:500), rabbit polyclonal VPS26 (Epitomics S1181, 1:1000) and VPS35 (Abcam 97545, 1:2000), mouse monoclonal GFP (Clones 7.1/13.1, Roche 11814460001, 1:5000), rat monoclonal MRP4 (Clone M4I-10, Abcam 15602, 1:250), rabbit polyclonal KIDINS220 (Proteintech 21856-1-ap, 1:1000), mouse monoclonal N-cadherin (Clone 13A9, Calbiochem CA1029, 1:1000), rabbit polyclonal EGFR (Cell Signalling Technologies, 2232L, 1:500), rabbit polyclonal MCT1 (gift from Dr. Andrew Halestrap, University of Bristol, 1:1000), rabbit monoclonal PDGFR $\beta$ (Clone Y92, Abcam 32570, 1:2000), rabbit polyclonal STEAP3 (Proteintech 17186-1-AP, 1:1000), rabbit polyclonal Glut1 (Abcam 15309, 1:100), rabbit polyclonal CSPG4 (Proteintech 55027-1-AP, 1:1000), mouse monoclonal TrailR1 (Clone DR-4-02, Aviva OASA01719, 1:100), rabbit monoclonal ItgB1 (Clone EO1042Y, Abcam, 52971, 1:2000), mouse monoclonal CD97 (Clone VIM3B, Biolegend 336302, 1:100), PTPRJ (Clone A3, Biolegend 328702, 1:100), mouse monoclonal ATP7A (Clone D9, Santa Cruz 376467, 1:100), mouse monoclonal TfnR (Clone H68.4, Invitrogen 13-6890, 1:1000), rabbit polyclonal Lamp1 (Abcam 24170, 1:800), mouse monoclonal Lamp1 (Fitzgerald, Clone H4A3, 1:300), mouse monoclonal HA-tag (Clone HAC5, Santa Cruz 57595, 1:100), mouse monoclonal Myc-tag (Clone 9E10, AbD Serotec, 1:250), sheep polyclonal Myc-tag (made in house for University of Bristol, 1:200), rabbit polyclonal RFP (MBL PM005, 1:2000).

\section{Western blotting}

Western blots were performed using standard procedures. Detection and quantification was done on a Licor Odyssey Infrared scanning system using fluorescently labelled secondary antibodies.

\section{SILAC interactome analysis}

All SILAC reagents were sourced from Thermo Fisher, only dialyzed FBS was from Sigma. Human RPE1 cells, HeLa cells or HEK293T cells were grown in the SILAC DMEM for at least 6 passages to achieve full labelling. GFP-SNX27 and GFP was either lentivirally 
transduced (HeLa and RPE1 cells) before the labelling or transiently transfected (HEK293T) after labelling. GFP expressing control cells were grown in unlabelled medium containing regular arginine and lysine (R0K0). GFP-SNX27 expressing cells were grown in medium containing 13C6-arginine and 4,4,5,5-D4-Lysine (R6K4). For the PDZ dependent interactome analysis in HEK293T cells, GFP was transiently expressed in unlabelled cells, GFP-SNX27 $\triangle$ PDZ was transfected into HEK293 cells grown in R6K4 and GFP-SNX27 was transfected into cells grown in medium containing 13C6-15N4-arginine and 13C6-15N2lysine (R10K8). Cells were lysed in precipitation buffer (50mM Tris-HCL, 0.5\% NP40, Roche Protease Inhibitor Cocktail) and GFP was precipitated with GFP-trap beads (Chromotek) for 30min. The beads were pooled, proteins were eluted in sample buffer, separated on Nupage 4-12\% precast gels (Invitrogen) and subjected to LC-MS-MS analysis on an Orbitrap Velos (Thermo) mass spectrometer. A detailed description of the mass spectrometric detection and quantification has been recently published ${ }^{3}$.

\section{Quantification of the surface proteome}

For the quantification of the surface proteome, Hela cells were grown in R0K0, R6K4 and R10K8 labelled DMEM for at least 6 doublings. For the first set of three experiments, one $60 \mathrm{~mm}$ dish of cells per condition was transfected with siRNA oligonucleotides against SNX27 (R6K4) and VPS35 (R0K0) as well as scrambled siRNA (R10K8) using Hiperfect reagent (Qiagen). For the second set of three experiments, SILAC isotopes were swapped between SNX27 and VPS35 suppressed cells and different siRNA oligonucleotides were used for the suppression. $72 \mathrm{~h}$ post transfection, cells were surface biotinylated with a commercially available kit (Piere/Thermo) according to the manufacturer's instructions. Cells were lysed in PBS with 2\% Triton-X100, lysates were pooled and streptavidin agarose (GE-Healthcare) was used to capture biotinylated membrane proteins. After capture, biotinylated proteins were washed extensively in $1.2 \mathrm{M} \mathrm{NaCl}$ containing PBS with $1 \%$ Triton-X100 to remove cytoskeletal proteins and contaminants from the transmembrane proteins, followed by elution of the proteins by boiling in DTT containing sample buffer. Proteins were then separated on Nupage precast gels, stained with colloidal coomassie stain (Invitrogen) and subjected to gel walking mass spectrometric analysis. For details of the mass spectrometric procedure see "SILAC interactome analysis" above.

SiRNA

For SNX27 suppression, the Dharmacon siGenome human smartpool (SNX27-1) or a single oligo with the published sequence ${ }^{5} 5^{\prime}$-CCAGGUAAUUGCAUUUGAA(dTdT)- $3^{\prime}$ (SNX27-2) was used. For VPS35 suppression, a mixture of either oligo 1 and 2 (VPS35-1) or oligo 3 and 4 (VPS35-2) of the on-target plus human smartpool (Dharmacon) were used. These were also the combinations used for the two sets of three experiments of the surface proteome quantification. SNX1, SNX2, SNX5 and SNX6 were suppressed with the siGenome smartpools (Dharmacon).

\section{Immunoprecipitations}

The respective GFP-tagged constructs were transfected into HEK293T cells using polyethylene-imine, and the cells were incubated for $48 \mathrm{~h}$, followed by lysis in either "native" IP buffer (50mM MES, 1\% w/v digitonin, $1 \mathrm{mM} \mathrm{MgAcetate,} 200 \mu \mathrm{M}$ sodium orthovanadate, 
$0.5 \mathrm{mM}$ EGTA, Roche protease inhibitor cocktail) or Tris based IP buffer (50mM Tris-HCL, $0.5 \%$ NP40 and Roche Protease inhibitor cocktail) and precipitation of GFP with GFP trap beads (Chromotek)

\section{Flow cytometry}

Flow cytometric detection of TrailR1, CD97 and PTPRJ was done as described previously for integrins ${ }^{3}$.

\section{Antibody uptake assays}

HeLa cells were transfected with siRNA against SNX27 and VPS35, split onto coverslips after $24 \mathrm{~h}$ and incubated for a further $48 \mathrm{~h}$. Medium was then replaced with full growth medium containing $100 \mu \mathrm{M}$ Leupeptin (Sigma) and $5 \mu \mathrm{g} / \mathrm{ml}$ of antibody against the extracellular domain of TrailR1 and CD97. The cells were incubated for 6h, allowing antibody to be taken up continuously. Cells were then washed, fixed and the antibody was visualized with an Alexa-488 coupled secondary antibody (Jackson) after permeabilization with $0.1 \%$ Saponin (Sigma). The cells were also stained for the lysosomal marker LAMP1 and colocalization was analyzed as described below.

\section{Direct recombinant interaction studies}

SNX1, VPS29-myc, VPS26-myc,VPS35, SNX27-FERM and SNX27-PDZ were cloned into pGEX and GST fusion proteins were purified from E-Coli using standard procedures. SNX27 domains were left on the beads as GST fusion proteins, whereas SNX1 and the VPS proteins were cleaved using precision protease (GE-Healthcare). To test for interactions, SNX1 and the VPS proteins were given into 50mM Tris-HCL, 200mM NaCl, $1 \%$ (v/v) Triton-X100 and $0.1 \%$ (w/v) BSA and incubated with the bead coupled SNX27 domains. After washing, bound proteins were eluted by boiling in SDS sample buffer and detected using western blotting.

\section{Degradation assays}

To measure degradation of surface proteins, HeLa cells were transfected with either control, SNX27, VPS35, SNX1 and SNX2 or SNX5 and SNX6 siRNA. 24h post transfection, before the effects of the siRNA were fully established, surface proteins were biotinylated with Sulfo-NHS-Biotin (Pierce/Thermo). 0, 12 and 24h after biotinylation (24, 36 and 48h post transfection of siRNA), cells were lysed, biotinylated proteins were captured with streptavidin agarose (GE-Healthcare) and detected by quantitative western blotting on an Odyssey scanner. Degradation was quantified as fluorescence signal remaining after 12 and $24 \mathrm{~h}$ in percent of signal intensity at timepoint 0 .

\section{SNX27 constructs and mutagenesis}

SNX27 was subdivided into its subdomains according to the domain boundaries proposed in the Uniprot (www.uniprot.org) database. The FERM-like domain was divided into the F1F3 subdomains according to a published model ${ }^{19}$. The $\Delta 67-77$ deletion was based upon a comparison of the crystal structure of NHERF1-PDZ domain 2 and the crystal structure of the SNX27 PDZ domain ${ }^{15}$. Using standard SDM procedures, amino acids 67-77 were 
excised to remove the divergent stretch and allow closure of the loop similar to the NHERF1-PDZ2 domain. siRNA resistant SNX27 was generated by introducing three silent base mis-matches (a1414c;11417a;a1420t) conferring resistance to siRNA SNX27-2 described above into the open reading frame.

\section{Rescue of Glut1 lysosomal mis-sorting with mutated SNX27 constructs}

Expression of endogenous SNX27 was suppressed in HeLa cells by transfecting siRNA SNX27-2. 24h after transfection, cells were trypsinized, seeded onto fibronectin (Sigma) coated coverslips and transfected with the respective GFP-tagged, siRNA resistant SNX27 constructs using Fugene 6 (Roche). $72 \mathrm{~h}$ after siRNA transfection, cells were fixed and stained for Lamp1 and Glut1. Rescue of lysosomal entry was visually scored using the following procedure: Cells in a randomly chosen field of vision were first scored for lysosomal accumulation of Glut1, followed by checking for expression of GFP tagged SNX27 proteins. At least 150 cells for each condition were scored. To confirm the findings, colocalization between Lamp1 and Glut1 signal was quantified over 60 individual cells from three independent experiments.

\section{Image acquisition and quantitation of colocalization}

All images were acquired on a Leica SP5 confocal system using the same settings across the respective conditions for each experiment. Following acquisition, images were analyzed with the Volocity software package (Perkin Elmer): To filter noise, thresholds were applied uniformly across conditions using the threshold tool of Volocity. After setting of the thresholds, the percentage of colocalization and the Pearson's correlation between the respective channels were quantified with the colocalization tool of the Volocity software. Each colocalization analysis is based upon the quantification of at least 30 images containing several hundred cells acquired in three independent experiments. For the Glut 1 rescue assay, 60 individual cells from three independent experiments were quantified for each condition shown. Statistical significance was determined by an unpaired t-test.

\section{Bioinformatic analysis of the surface proteome data}

The data sets from the six independent experiments were combined into a single MySQL table and all subsequent analysis was performed using a PHP script to address the database. To impose a normal distribution on the data set, which is a prerequisite for t-test analysis, the individual heavy/light and heavy/medium ratios were converted to their Log2 equivalents and all further analysis was performed on these data. The mean and SD was calculated for each of the 10,156 accessions in the dataset. The population mean for all accession was also calculated and this figure was used to normalise the dataset by subtracting this value from each of the accession means: thus, proteins that were not differentially expression between the treatments would have mean values close to 0 while those up- and down-regulated would have positive and negative values, respectively. One sample t-tests were then performed for each of the accessions and $p$-values (two sided t-test) calculated for each. 


\section{Radioactive glucose uptake assay}

Glucose uptake assays were performed as essentially as previously described ${ }^{34}$. In brief, HeLa cells were washed with HEPES buffered saline (HBS) buffer (20mM HEPES pH 7.4, $140 \mathrm{mM} \mathrm{NaCl}, 5 \mathrm{mM} \mathrm{KCl}, 2.5 \mathrm{mM} \mathrm{MgSO}_{4}, 1 \mathrm{mM} \mathrm{CaCl}_{2}$ ) prior to initiation of the assay with addition of HBS containing 1uCi 2-deoxy-D- $\left[{ }^{3} \mathrm{H}\right]$-glucose $(8 \mathrm{Ci} / \mathrm{mmol}$; Perkin Elmer) and $50 \mu \mathrm{M}$ unlabelled 2-deoxy-D-glucose. After $10 \mathrm{~min}$ uptake at $37^{\circ} \mathrm{C}$, cells were washed in PBS, solubilized in $1 \%$ Triton $\mathrm{X}-100$ and ${ }^{3} \mathrm{H}$ was quantitated by scintillation counting. Nonspecific glucose uptake was determined in the presence of cytochalasin B (10 $\mu \mathrm{M}$; Sigma).

\section{Statistical analysis}

All quantified western blot and confocal colocalization data are the mean of the indicated number of independent experiments. The raw data from the quantitative western blotting were first normalized to percent of control (comparative western blots) or to percent of timepoint t0 (degradation assays) for each condition. Mean and standard error over the indicated number of independent experiments were calculated, followed by an unpaired teacher's t-test to determine statistical significance. Colocalization data from at least three independent experiments was averaged across individual experiments and standard deviation was calculated across the three experiments. An unpaired teacher's t-test was then used to analyze statistical significance. For all statistical tests, a $p=v a l u e<0.05$ was considered significant and is indicated by *. The individual data points for all experiments with statistics are shown in the statistics source data excel file.

\section{Supplementary Material}

Refer to Web version on PubMed Central for supplementary material.

\section{Acknowledgments}

FS is a Royal Society Newton Fellow. MG and AJB are supported by Wellcome Trust 4 Year PhD Studentships awarded through the Dynamic Cell Biology programme (083474). PJC is supported by the Wellcome Trust (089928 and 085743) and the Royal Society. We thank Mark Bass and David Stephens for critical reading of the manuscript.

\section{References}

1. Cullen PJ. Endosomal sorting and signalling: an emerging role for sorting nexins. Nat Rev Mol Cell Biol. 2008; 9:574-582. doi: 10.1038/nrm2427 ; pii: nrm2427. [PubMed: 18523436]

2. van Kerkhof $P$, et al. Sorting nexin 17 facilitates LRP recycling in the early endosome. EMBO J. 2005; 24:2851-2861. doi: 10.1038/sj.emboj.7600756 ; pii: 7600756. [PubMed: 16052210]

3. Steinberg F, Heesom KJ, Bass MD, Cullen PJ. SNX17 protects integrins from degradation by sorting between lysosomal and recycling pathways. J Cell Biol. 2012; 197:219-230. doi: 10.1083/ jcb.201111121 ; pii: jcb.201111121. [PubMed: 22492727]

4. Bottcher RT, et al. Sorting nexin 17 prevents lysosomal degradation of beta1 integrins by binding to the beta1-integrin tail. Nat Cell Biol. 2012; 14:584-592. doi: 10.1038/ncb2501; pii: ncb2501. [PubMed: 22561348]

5. Lauffer BE, et al. SNX27 mediates PDZ-directed sorting from endosomes to the plasma membrane. J Cell Biol. 2010; 190:565-574. doi: 10.1083/jcb.201004060 ; pii: jcb.201004060. [PubMed: 20733053] 
6. Temkin P, et al. SNX27 mediates retromer tubule entry and endosome-to-plasma membrane trafficking of signalling receptors. Nat Cell Biol. 2011; 13:715-721. doi: 10.1038/ncb2252 ; pii: ncb2252. [PubMed: 21602791]

7. Arighi CN, Hartnell LM, Aguilar RC, Haft CR, Bonifacino JS. Role of the mammalian retromer in sorting of the cation-independent mannose 6-phosphate receptor. J Cell Biol. 2004; 165:123-133. doi: 10.1083/jcb.200312055 ; pii: jcb.200312055. [PubMed: 15078903]

8. Carlton J, et al. Sorting nexin-1 mediates tubular endosome-to-TGN transport through coincidence sensing of high-curvature membranes and 3-phosphoinositides. Curr Biol. 2004; 14:1791-1800. doi: 10.1016/j.cub.2004.09.077 ; pii: S096098220400750X. [PubMed: 15498486]

9. Seaman MN. Cargo-selective endosomal sorting for retrieval to the Golgi requires retromer. J Cell Biol. 2004; 165:111-122. doi: 10.1083/jcb.200312034 ; pii: jcb.200312034. [PubMed: 15078902]

10. Wassmer T, et al. A loss-of-function screen reveals SNX5 and SNX6 as potential components of the mammalian retromer. J Cell Sci. 2007; 120:45-54. doi: 10.1242/jcs.03302 ; pii: jcs.03302. [PubMed: 17148574]

11. Wassmer $\mathrm{T}$, et al. The retromer coat complex coordinates endosomal sorting and dynein-mediated transport, with carrier recognition by the trans-Golgi network. Dev Cell. 2009; 17:110-122. doi: 10.1016/j.devcel.2009.04.016 ; pii: S1534-5807(09)00178-6. [PubMed: 19619496]

12. Trinkle-Mulcahy $\mathrm{L}$, et al. Identifying specific protein interaction partners using quantitative mass spectrometry and bead proteomes. J Cell Biol. 2008; 183:223-239. doi: 10.1083/jcb.200805092 ; pii: jcb.200805092. [PubMed: 18936248]

13. Rincon E, et al. Proteomics identification of sorting nexin 27 as a diacylglycerol kinase zetaassociated protein: new diacylglycerol kinase roles in endocytic recycling. Mol Cell Proteomics. 2007; 6:1073-1087. doi: 10.1074/mcp.M700047-MCP200 ; pii: M700047-MCP200. [PubMed: 17351151]

14. Valdes JL, et al. Sorting nexin 27 protein regulates trafficking of a p21-activated kinase (PAK) interacting exchange factor (beta-Pix)-G protein-coupled receptor kinase interacting protein (GIT) complex via a PDZ domain interaction. J Biol Chem. 2011; 286:39403-39416. doi: 10.1074/ jbc.M111.260802 ; pii: M111.260802. [PubMed: 21926430]

15. Balana B, et al. Mechanism underlying selective regulation of $\mathrm{G}$ protein-gated inwardly rectifying potassium channels by the psychostimulant-sensitive sorting nexin 27. Proc Natl Acad Sci U S A. 2011; 108:5831-5836. doi: 10.1073/pnas.1018645108 ; pii: 1018645108. [PubMed: 21422294]

16. Setty SR, et al. Cell-specific ATP7A transport sustains copper-dependent tyrosinase activity in melanosomes. Nature. 2008; 454:1142-1146. doi: 10.1038/nature07163 ; pii: nature07163. [PubMed: 18650808]

17. Zhao FQ, Keating AF. Functional properties and genomics of glucose transporters. Curr Genomics. 2007; 8:113-128. [PubMed: 18660845]

18. Traer CJ, et al. SNX4 coordinates endosomal sorting of TfnR with dynein-mediated transport into the endocytic recycling compartment. Nat Cell Biol. 2007; 9:1370-1380. doi: 10.1038/ncb1656 ; pii: ncb1656. [PubMed: 17994011]

19. Ghai R, et al. Phox homology band 4.1/ezrin/radixin/moesin-like proteins function as molecular scaffolds that interact with cargo receptors and Ras GTPases. Proc Natl Acad Sci U S A. 2011; 108:7763-7768. doi: 10.1073/pnas.1017110108 ; pii: 1017110108. [PubMed: 21512128]

20. Harbour ME, Breusegem SY, Seaman MN. Recruitment of the endosomal WASH complex is mediated by the extended 'tail' of Fam 21 binding to the retromer protein Vps35. Biochem J. 2012; 442:209-220. doi: 10.1042/BJ20111761 ; pii: BJ20111761. [PubMed: 22070227]

21. Jia D, Gomez TS, Billadeau DD, Rosen MK. Multiple repeat elements within the FAM21 tail link the WASH actin regulatory complex to the retromer. Mol Biol Cell. 2012; 23:2352-2361. doi: 10.1091/mbc.E11-12-1059 ; pii: mbc.E11-12-1059. [PubMed: 22513087]

22. Harbour ME, et al. The cargo-selective retromer complex is a recruiting hub for protein complexes that regulate endosomal tubule dynamics. J Cell Sci. 2010; 123:3703-3717. doi: 10.1242/jcs. 071472 ; pii: jcs.071472. [PubMed: 20923837]

23. Shi H, Rojas R, Bonifacino JS, Hurley JH. The retromer subunit Vps26 has an arrestin fold and binds Vps35 through its C-terminal domain. Nat Struct Mol Biol. 2006; 13:540-548. doi: 10.1038/ nsmb1103 ; pii: nsmb1103. [PubMed: 16732284] 
24. Hayashi $\mathrm{H}$, et al. Sorting nexin 27 interacts with multidrug resistance-associated protein 4 (MRP4) and mediates internalization of MRP4. J Biol Chem. 2012; 287:15054-15065. doi: 10.1074/ jbc.M111.337931 ; pii: M111.337931. [PubMed: 22411990]

25. Seaman MN, Harbour ME, Tattersall D, Read E, Bright N. Membrane recruitment of the cargoselective retromer subcomplex is catalysed by the small GTPase Rab7 and inhibited by the RabGAP TBC1D5. J Cell Sci. 2009; 122:2371-2382. doi: 10.1242/jcs.048686 ; pii: jcs.048686. [PubMed: 19531583]

26. Elkins JM, et al. Structure of PICK1 and other PDZ domains obtained with the help of self-binding C-terminal extensions. Protein Sci. 2007; 16:683-694. doi: 10.1110/ps.062657507 ; pii: 16/4/683. [PubMed: 17384233]

27. Bunn RC, Jensen MA, Reed BC. Protein interactions with the glucose transporter binding protein GLUT1CBP that provide a link between GLUT1 and the cytoskeleton. Mol Biol Cell. 1999; 10:819-832. [PubMed: 10198040]

28. Wieman HL, et al. An essential role for the Glut1 PDZ-binding motif in growth factor regulation of Glut1 degradation and trafficking. Biochem J. 2009; 418:345-367. doi: 10.1042/BJ20081422 ; pii: BJ20081422. [PubMed: 19016655]

29. Puthenveedu MA, et al. Sequence-dependent sorting of recycling proteins by actin-stabilized endosomal microdomains. Cell. 2010; 143:761-773. doi: 10.1016/j.cell.2010.10.003 ; pii: S0092-8674(10)01139-6. [PubMed: 21111236]

30. Gomez TS, Gorman JA, de Narvajas AA, Koenig AO, Billadeau DD. Trafficking defects in WASH-knockout fibroblasts originate from collapsed endosomal and lysosomal networks. Mol Biol Cell. 2012; 23:3215-3228. doi: 10.1091/mbc.E12-02-0101 ; pii: mbc.E12-02-0101. [PubMed: 22718907]

31. Ghai R, et al. Structural basis for endosomal trafficking of diverse transmembrane cargos by PXFERM proteins. Proc Natl Acad Sci U S A. 2013 doi: 10.1073/pnas.1216229110 ; pii: 1216229110.

32. Zech T, et al. The Arp $2 / 3$ activator WASH regulates alpha5beta1-integrin-mediated invasive migration. J Cell Sci. 2011; 124:3753-3759. doi: 10.1242/jcs.080986 ; pii: jcs.080986. [PubMed: 22114305] 

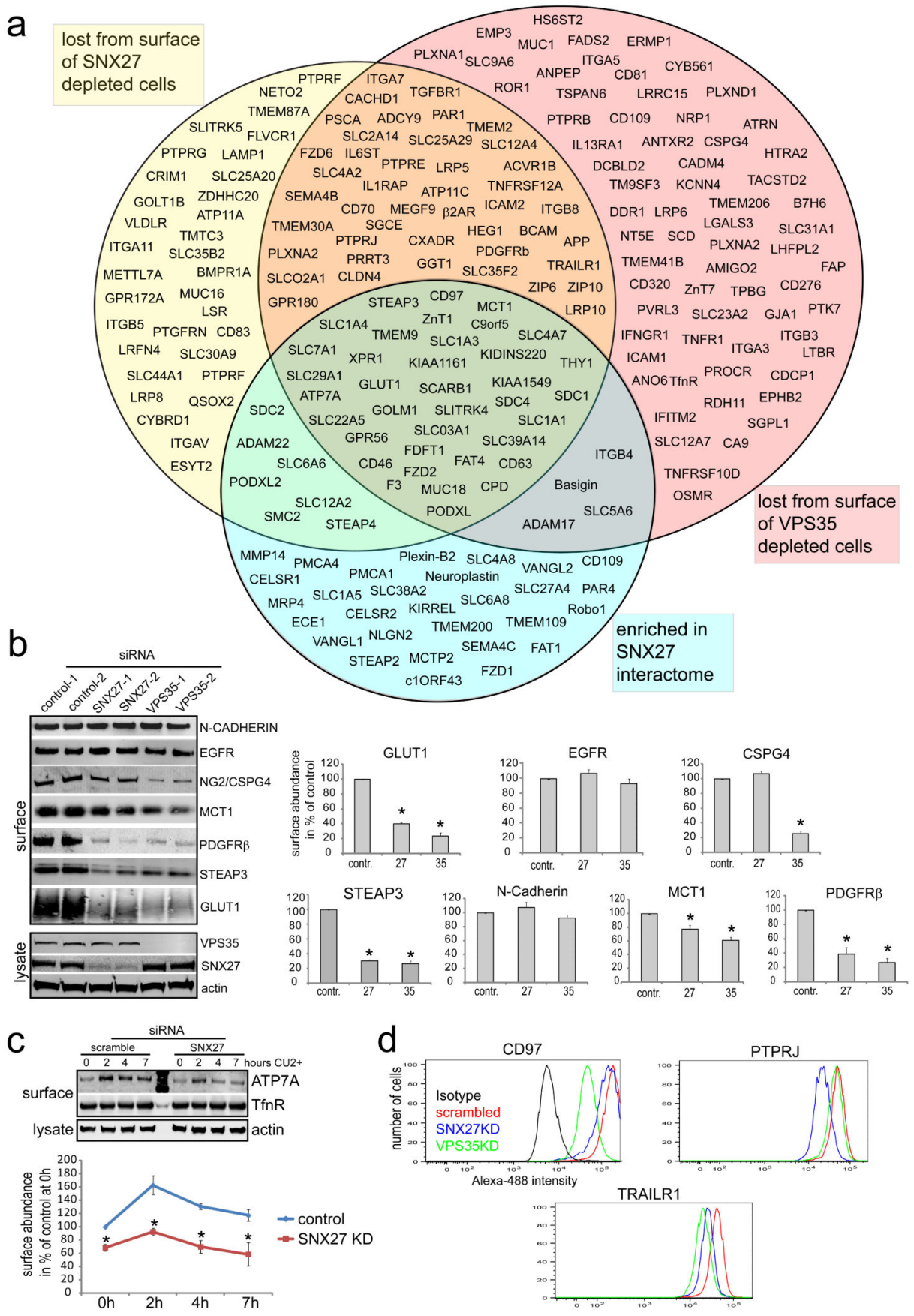

Fig. 1. Interactome analysis combined with a quantitative analysis of the surface proteome of SNX27 and VPS35 depleted Hela cells uncovers transmembrane cargo for SNX27 and retromer (a) HeLa cells were grown three different SILAC media, SNX27 and VPS35 expression was suppressed with siRNA and the biotinylated surface proteome was quantified compared to control cells. Proteins in the yellow circle were lost at least 1.4 fold from SNX27 suppressed cells while the red circle contains proteins lost at least 1.4 fold from the surface of VPS35 deficient cells. The blue circle contains proteins that were at least 3 fold enriched in a SILAC based SNX27 interactome analysis. Hence, proteins in the lower overlap region of the three circles interact with SNX27 and are lost from the surface of SNX27 and VPS35 
depleted cells, which makes them high confidence new cargo for the SNX27 and retromer. (b) Validation of selected hits by fluorescence based quantitative western blotting on an odyssey scanner. SNX27 and VPS35 were suppressed with two individual oligos and surface fractions were analyzed for the abundance of the indicated proteins. The quantifications show the mean of least three independent experiments ( $\mathrm{n}=3$ for EGFR and CSPG4, $\mathrm{n}=4$ for $\mathrm{N}$-Cadherin and MCT1, $\mathrm{n}=5$ for GLUT1, $\mathrm{n}=6$ for PDGFR $\beta$ ) * indicates $\mathrm{p}<0.05$, unpaired t-test, error bars $=$ s.e.m., individual data points shown in statistics source data, uncropped blots in Fig S5) (c) ATP7A surface levels were analyzed under copper induced $(200 \mu \mathrm{M} \mathrm{CuCl} 2)$ conditions in SNX control and SNX27 depleted cells over a timecourse of $7 \mathrm{~h}$ (graph represents the mean of three independent experiments, *indicates $\mathrm{p}<0.05$, unpaired t-test, error bars $=$ s.e.m., individual data points shown in statistics source data) (d) Flow cytometric analysis of surface resident CD97, PTPRJ and TrailR1 in SNX27 and VPS35 deficient cells 

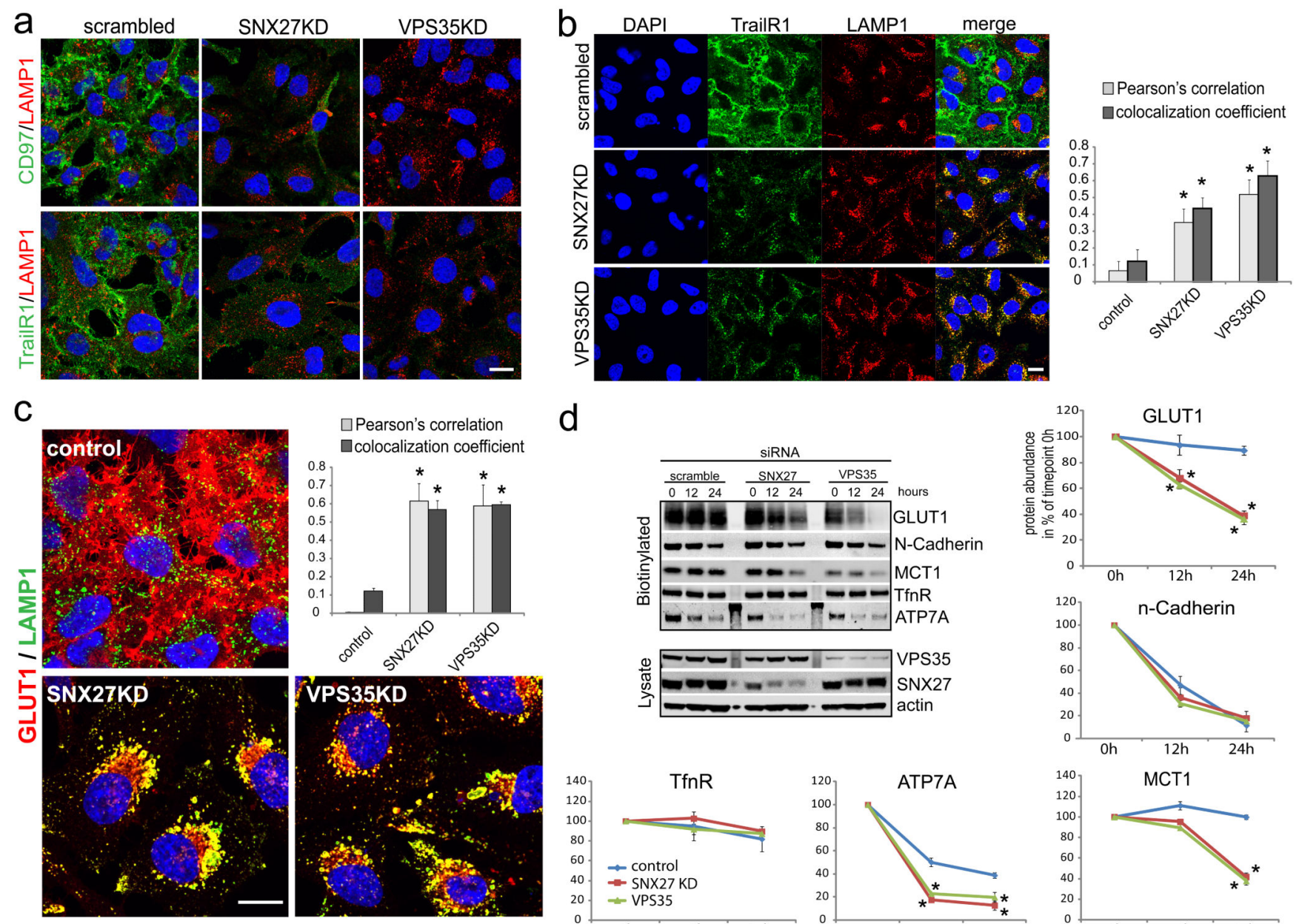

d
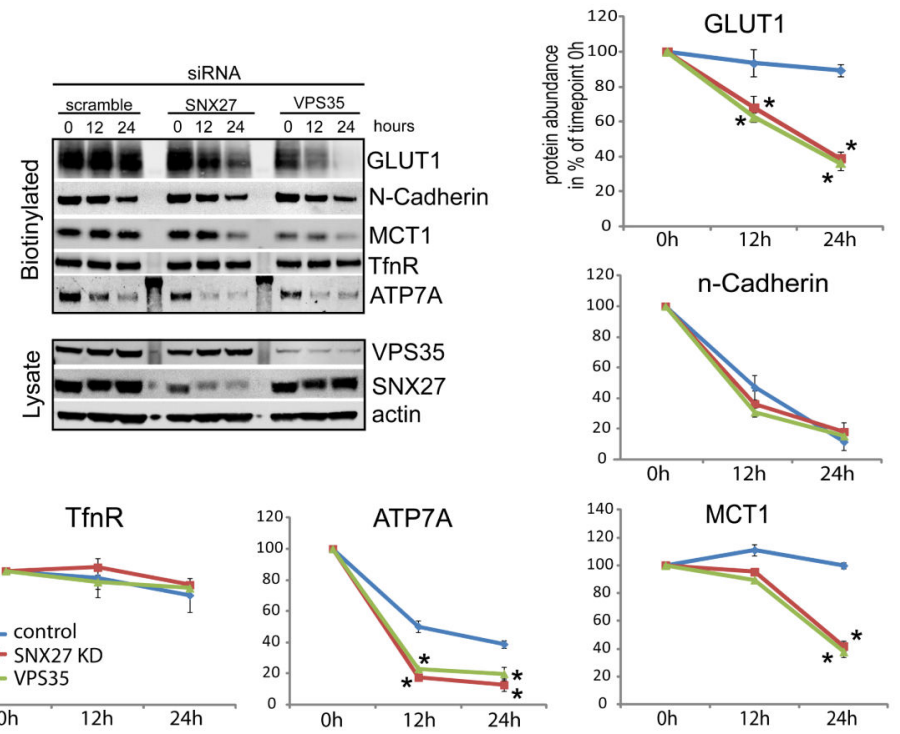

Fig. 2. SNX27-retromer cargo is mis-sorted into lysosomes and degraded in the absence of SNX27 or VPS35

(a) Immunofluorescent analysis of CD97 and TrailR1 in SNX27 and VPS35 depleted HeLa cells (green) with lysosomes stained with a LAMP1 antibody (red). (b) SNX27 and VPS35 suppressed cells were incubated with the lysosomal protease inhibitor Leupeptin $(100 \mu \mathrm{M})$ and antibodies against the ectodomain of TrailR1 and CD97 for 6h, followed by fixing, permeabilization and staining of lysosomes (LAMP1) and the internalized antibodies. Colocalization of internalized antibody and LAMP1 was quantified, the graph represents the mean of three independent experiments with 10 images each $(n=3, *$ indicates $p<0.05$, unpaired t-test; error bars $=$ s.d., scale bar $=10 \mu \mathrm{m}$, individual data points shown in statistics source data) (c) Immunofluorescent staining and colocalization analysis of endogenous Glut1 glucose transporter and lysosomal marker LAMP1 in SNX27 and VPS35 deficient Hela cells. The graph represents the mean of three independent experiments with 10 images each. $(\mathrm{n}=3$, * indicates $\mathrm{p}<0.05$, unpaired t-test; error bars $=$ s.d., scale bar $=10 \mu \mathrm{m}$, individual data points shown in statistics source data) (d) Degradation assays of biotin labeled SNX27retromer cargo. HeLa cells were transfected with siRNA against SNX27 and VPS35 and surface biotinylated $24 \mathrm{~h}$ post transfection, before the effects of suppression became fully evident. Biotinylated proteins were captured from lysates with streptavidin beads at 
indicated timepoints after biotinylation and subjected to quantitative western blotting on an odyssey scanner. The plots represent the mean of 3 (ATP7A, GLUT1, TfnR, MCT1) or 4 $(\mathrm{N}-\mathrm{Cadherin})$ independent experiments. $(*$ indicates $\mathrm{p}<0.05$, unpaired $\mathrm{t}$-test; error bars $=$ s.e.m., individual data points shown in statistics source data, uncropped blots in Fig S5) 
a
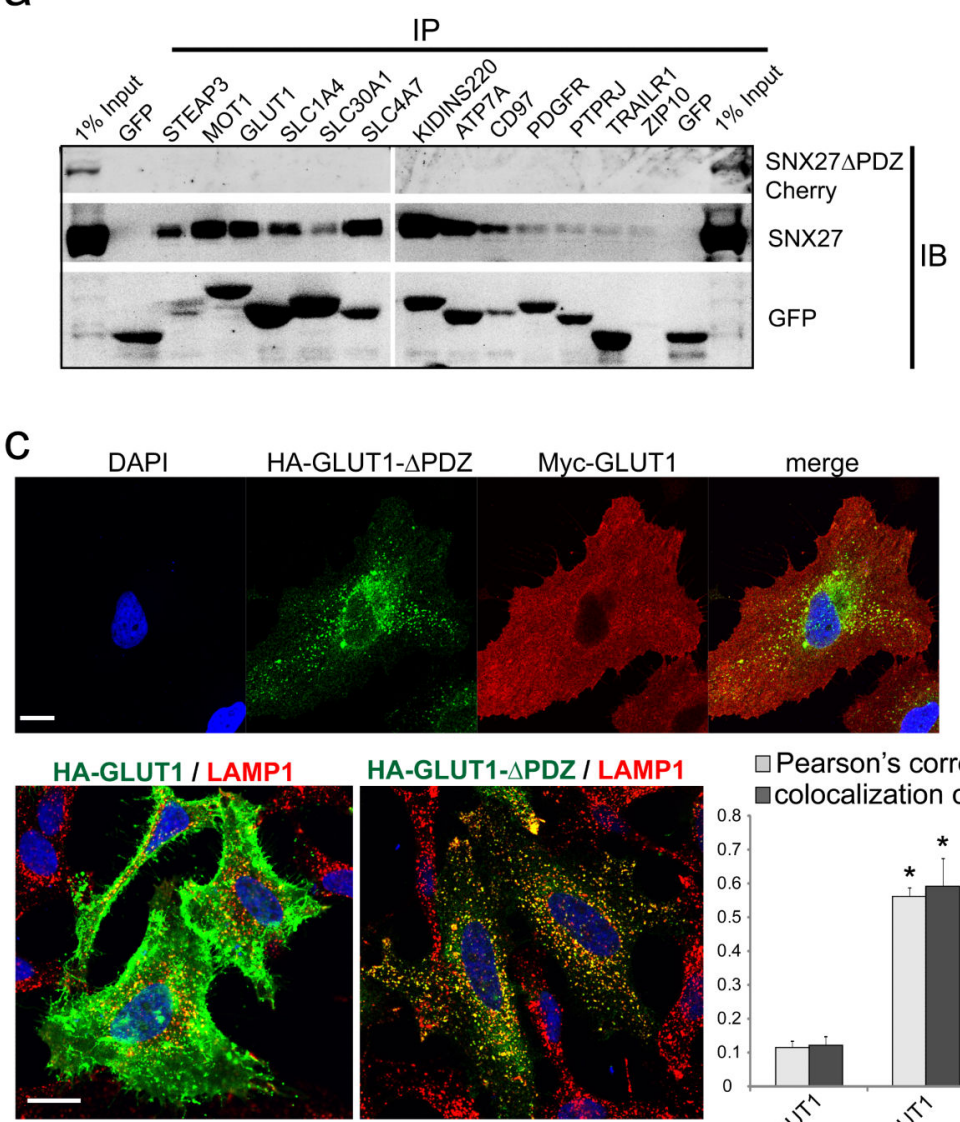

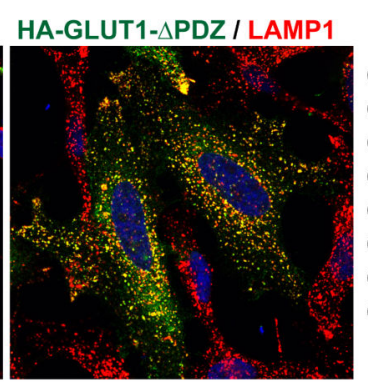

b

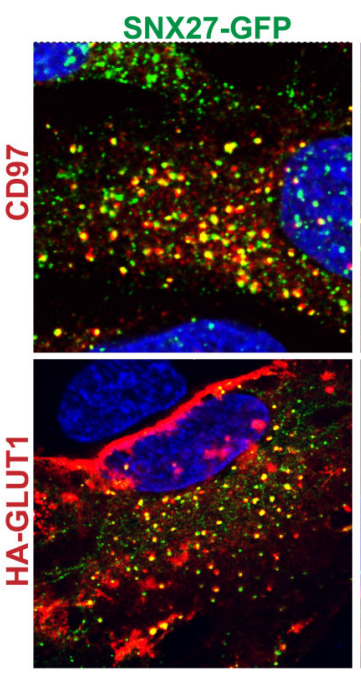

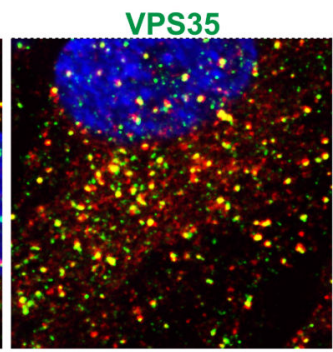

d
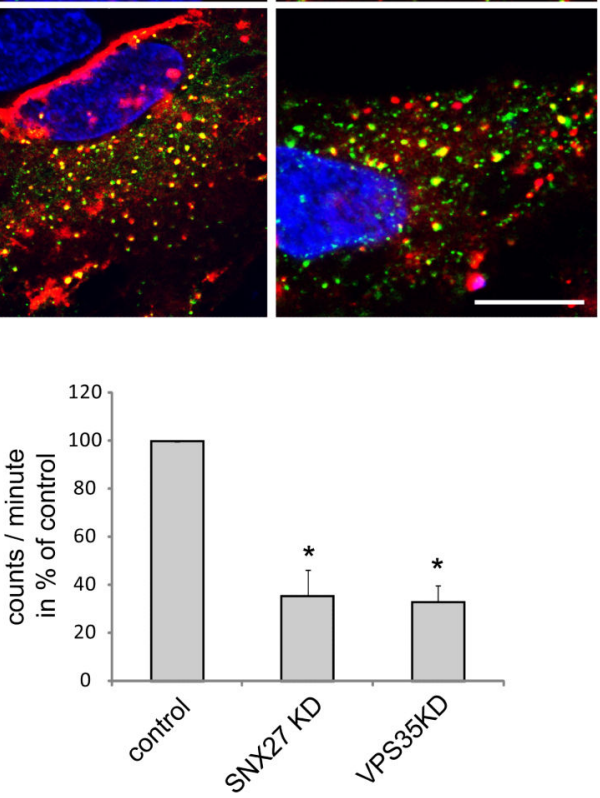

Fig. 3. PDZ interaction motifs at the C-terminus of membrane proteins interact with SNX27 and are required to maintain surface levels

(a) The cytoplasmic tails of selected proteins from the surface proteome analysis were fused to GFP and analyzed for binding of endogenous SNX27 and mCherry-tagged SNX27 $\triangle$ PDZ in immunoprecipitation experiments (uncropped blots shown in Fig. S5) (b) Antibody uptake experiments with antibodies against endogenous CD97 and HA-tagged Glut1. After $1 \mathrm{~h}$ incubation at $37^{\circ}$, internalized Glut 1 and CD97co-localized extensively with endogenous VPS35 or GFP-SNX27 on vesicular structures. (c) Upper panel: Immunofluorescent staining of HA-tagged Glut1 full length and Myc-tagged Glut1 with a truncated PDZ binding motif

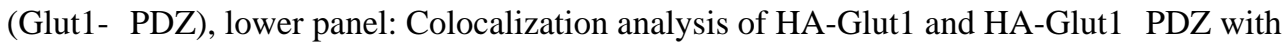
the lysosomal marker LAMP1. The graph represents the mean of three independent experiments with 10 images each. $(n=3$, * indicates $\mathrm{p}<0.05$, unpaired t-test; error bars $=$ s.d., individual data points shown in statistics source data, scale bar $=10 \mu \mathrm{m})(\mathrm{d})$ Uptake assay with radioactive glucose in SNX27 and VPS35 depleted cells. The graph represents the mean of four independent experiments done in quadruplicates. $\left(n=4,{ }^{*}\right.$ indicates $\mathrm{p}<0.05$, unpaired t-test; error bars = s.e.m., individual data points shown in statistics source data) 

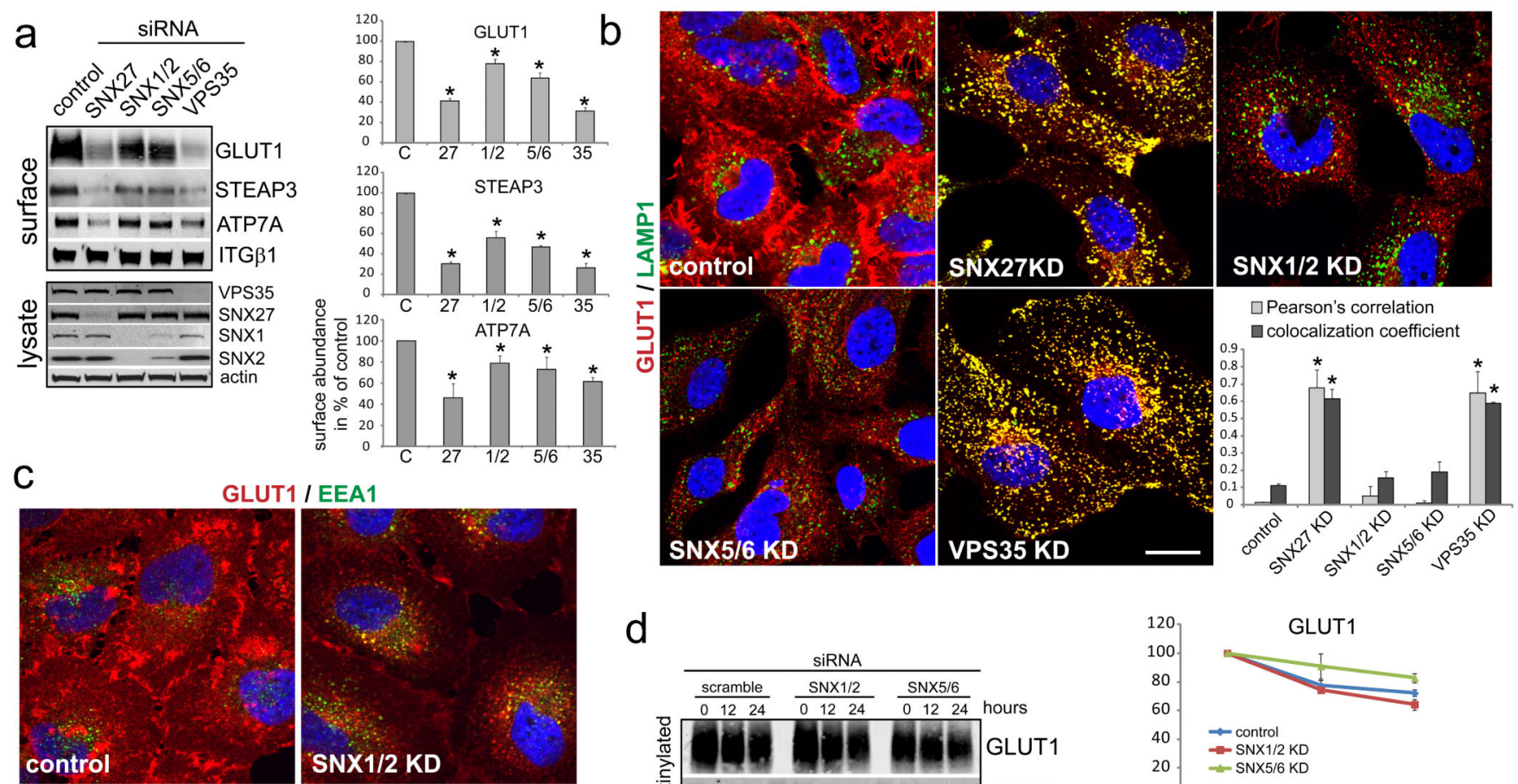

d
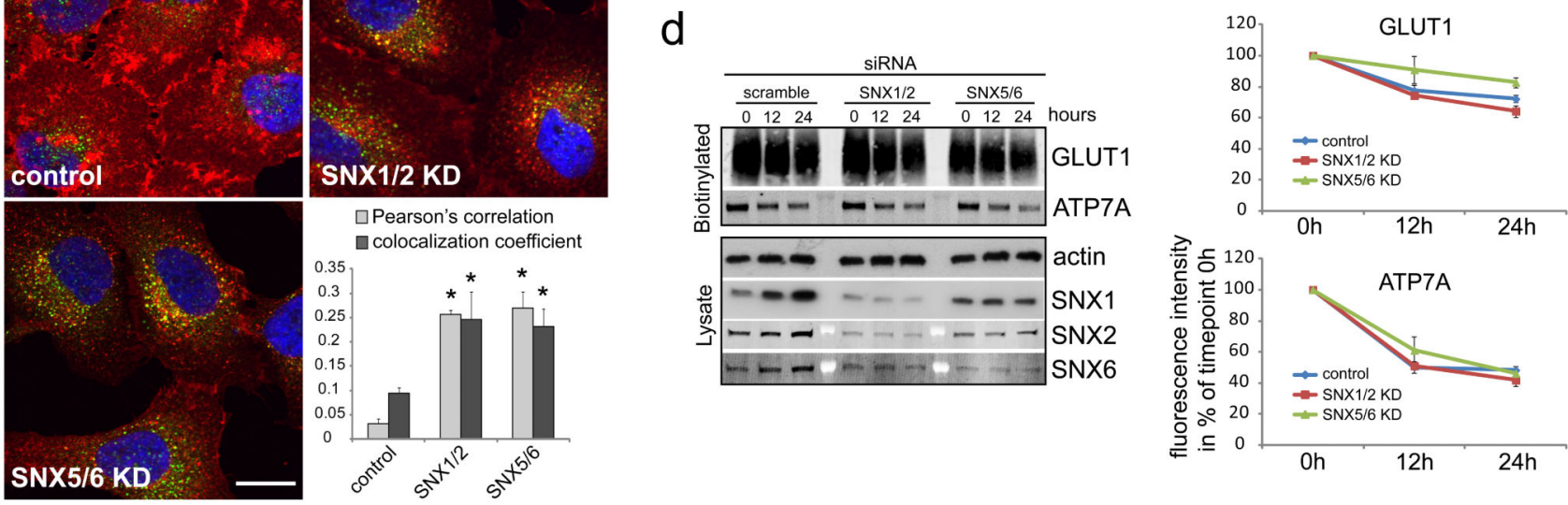

Fig. 4. Suppression of retromer SNX-BAR proteins does not phenocopy SNX27 and VPS35 loss of function but appears to cause trafficking delays

(a) HeLa cells were transfected with the indicated siRNAs and surface abundance of Glut1, ATP7A and Steap3 was determined by quantitative western blotting (graph represents the mean of 3 (ATP7A), 6 (GLUT1) and 5 (STEAP3) independent experiments, * indicates $\mathrm{p}<0.05$, unpaired t-test; error bars $=$ s.e.m., individual data points shown in statistics source data, uncropped blots shown in Fig. S5) (b) Immunofluorescent colocalization analysis of endogenous Glut1 and LAMP1 in SNX27-retromer and SNX-BAR depleted cells. The graph represents the mean of 30 images acquired in three independent experiments $(n=3$, * indicates $\mathrm{p}<0.05$, unpaired t-test; error bars $=$ s.d., Scale bar $=10 \mu \mathrm{m}$, individual data points shown in statistics source data) (c) Colocalization analysis of endogenous Glut1 and the early endosome marker EEA1 in SNX-BAR depleted cells. The graph represents the mean of three independent experiments with 10 images each. $(n=3, *$ indicates $p<0.05$, unpaired $t$ test; error bars $=$ s.d., Scale bar $=10 \mu \mathrm{m}$, individual data points shown in statistics source data) (d) Mean of four degradation assays of Glut1 and ATP7A in SNX-BAR depleted cells (for assay details see Fig. 2D or methods, $\mathrm{n}=4$, * indicates $\mathrm{p}<0.05$, unpaired t-test; error bars = s.e.m., individual data points shown in statistics source data, uncropped blots shown in Fig. S5) 
a

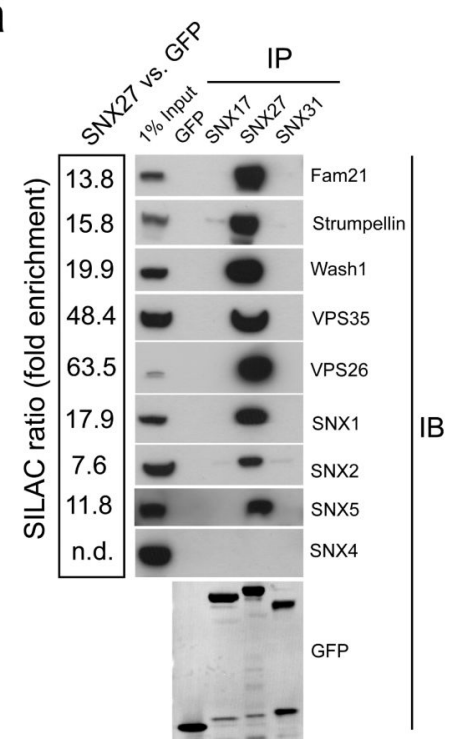

b
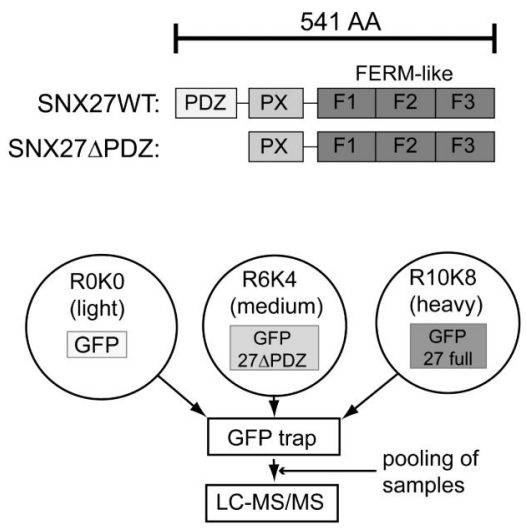

C

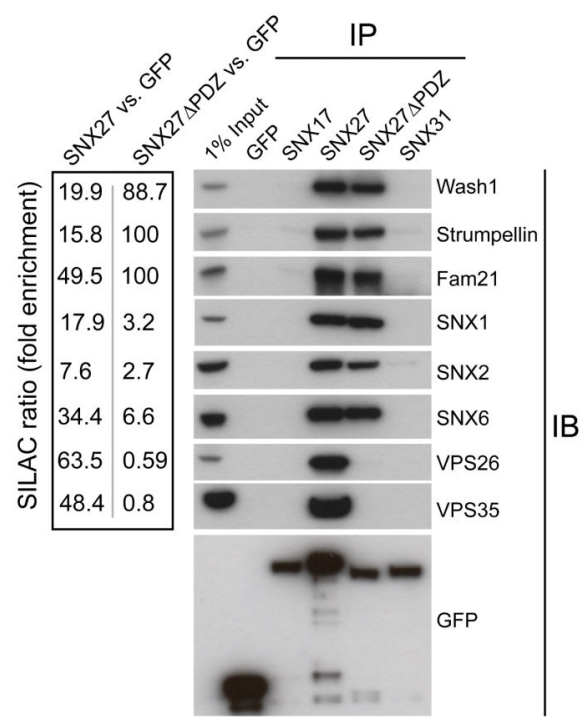

Fig. 5. SILAC and western blot analysis of the SNX27 interactome reveals an interaction of SNX27 with all retromer components

(a) SILAC based comparative proteomic analysis of GFP-trap precipitated GFP-SNX27 versus GFP. GFP-SNX27 and GFP were lentivirally expressed in human RPE1 cells, the cells were grown in normal (GFP) and heavy isotope labeled (SNX27) medium, followed by lysis and precipitation with GFP-trap beads. Precipitates were pooled, separated by SDSPAGE and subjected to gel walking LC-MS-MS analysis on an orbitrap mass spectrometer. The SILAC ratio is the fold-enrichment of proteins in GFP-SNX27 precipitates over GFP. Components of the SNX-BAR retromer as well as WASH complex components were highly enriched. Western blots of precipitates from HEK293 cells confirmed the SILAC results (uncropped blots shown in Fig. S5). (b) Schematic overview of SNX27 and SNX27 $\triangle$ PDZ domain structure (top) and triple SILAC setup to obtain the PDZ dependent interactome of SNX27 (bottom) (c) Triple SILAC and western blot analysis of transiently transfected GFPSNX27 vs. GFP-SNX27 $\triangle$ PDZ vs. GFP alone in HEK293 cells. The SILAC ratios indicated that the trimeric VPS complex precipitated through the PDZ domain, whereas WASH complex and SNX-BAR proteins precipitated through another domain. Western blots confirmed these findings (uncropped blots shown in Fig. S5). 
a

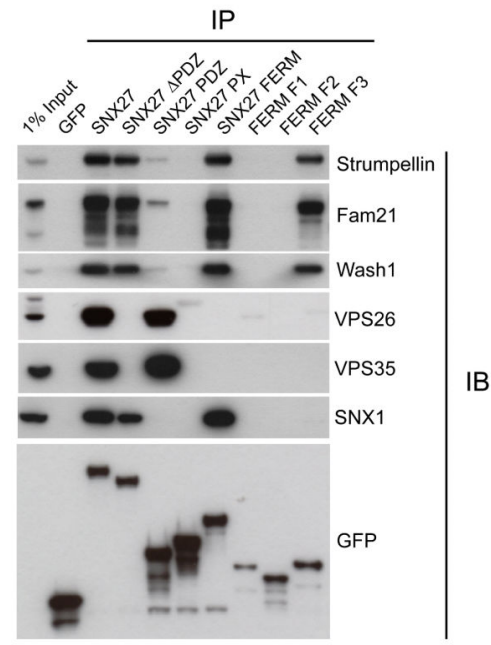

C

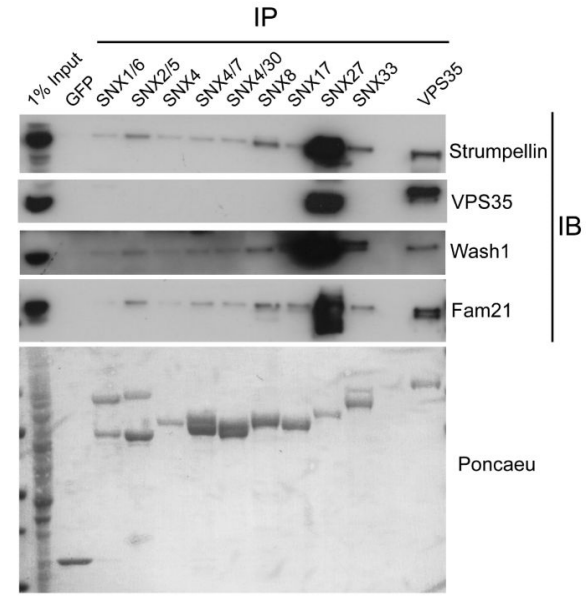

b

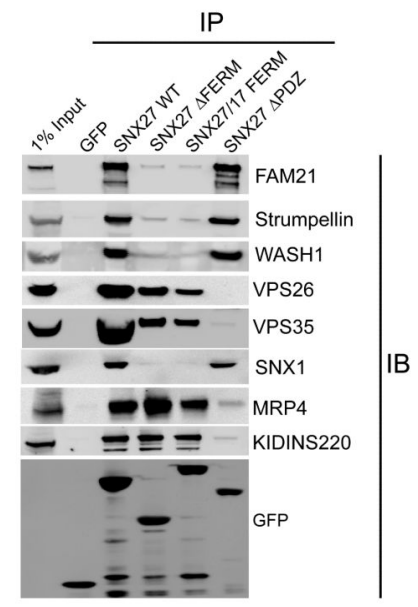

d
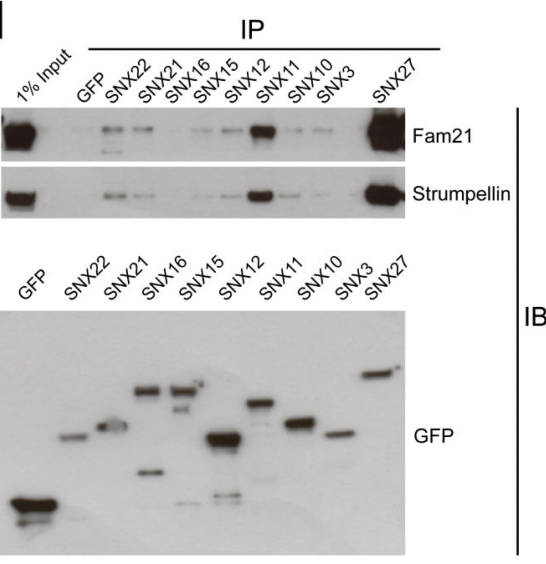

e
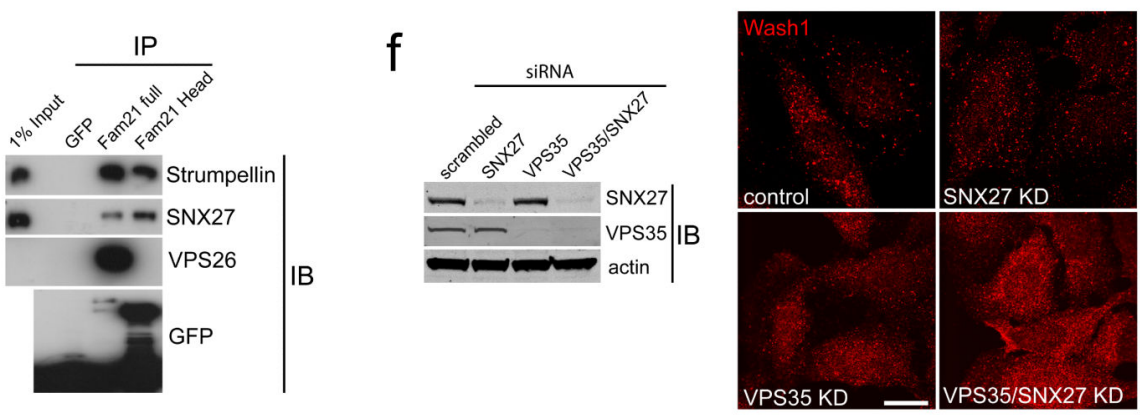

Fig. 6. The SNX27 FERM like domain interacts with the WASH complex independently of the retromer VPS26:29:35 complex

If not otherwise indicated, interactions were analyzed through GFP-trap precipitations from transiently transfected HEK293T cells (a) SNX27 was split into the indicated GFP fused sub-domains, which were analyzed for WASH complex, SNX1, VPS26 and VPS35 binding. (b) The indicated GFP fusion constructs were analyzed for binding to WASH and retromer complexes as well as for binding to the PDZ ligand bearing transmembrane proteins MRP4 and KIDINS220. (c) and (d) The indicated GFP-tagged sorting nexins were analyzed for binding to the WASH complex and retromer. (e) YFP-Fam21 and and GFP-Fam21-Head 
were analyzed for binding to endogenous retromer complex (VPS26), WASH complex (Strumpellin) and endogenous SNX27. (f) SNX27 and VPS35 were depleted from HeLa cells with siRNA and Wash1 was visualized by immunofluorescent staining. Scale bar = $10 \mu \mathrm{m}$. All uncropped blots shown in Fig. S6. 
a

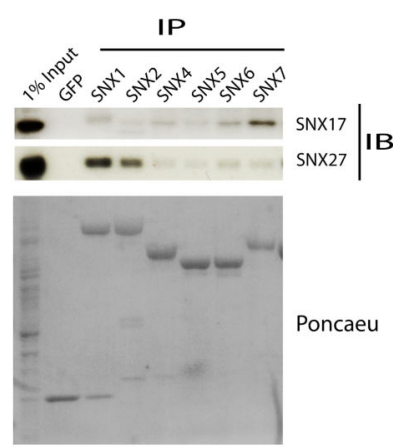

e

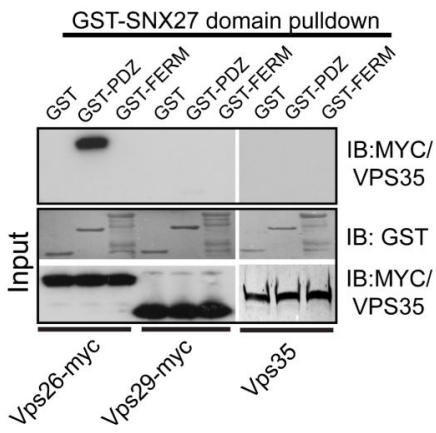

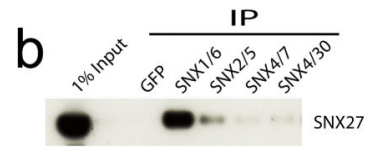

C

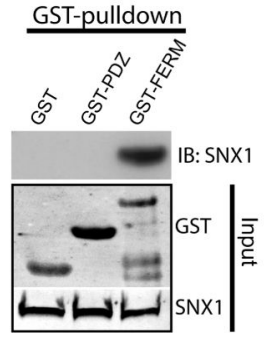

d

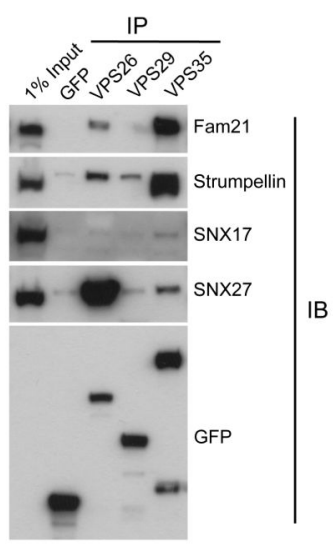

f

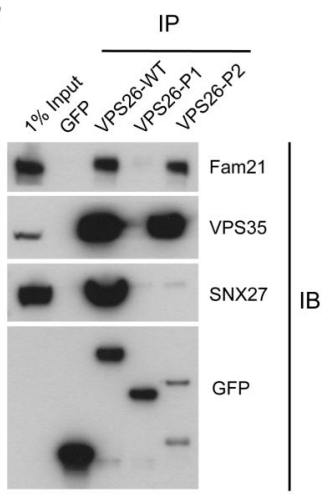

g

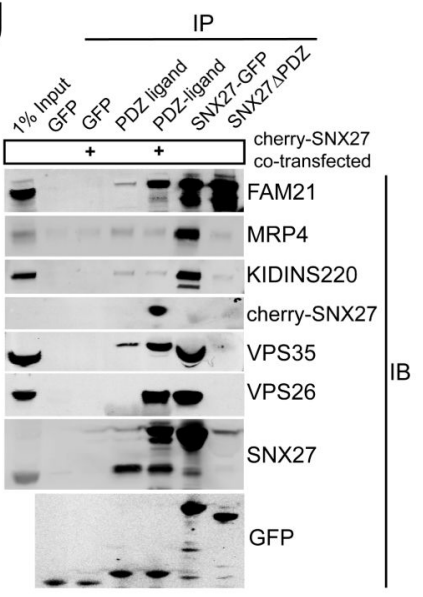

h

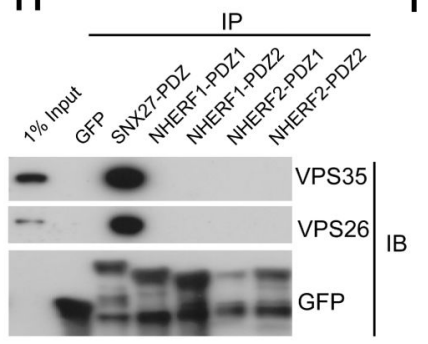

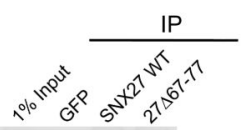

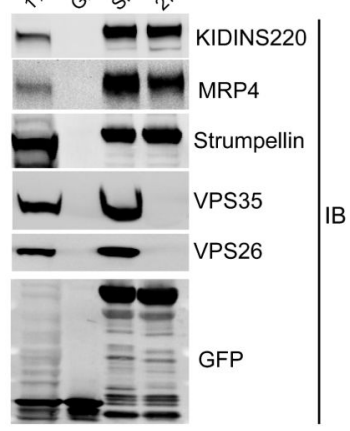

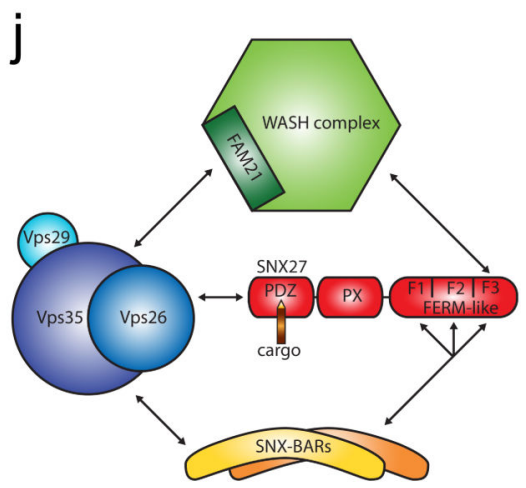

Fig. 7. Analysis of the SNX27-retromer interaction

If not otherwise indicated, interactions were analyzed through GFP-trap precipitations from transiently transfected HEK293T cells (a) and (b) The indicated GFP-tagged sorting nexins were analyzed for binding to SNX27 and SNX17. (c) Interaction analysis with recombinant SNX1 and GST-tagged SNX27 PDZ domain and FERM-like domain. (d) GFP-tagged VPS26, VPS29 and VPS35 were analyzed for binding to SNX17 and SNX27 as well as to WASH complex components FAM21 and Strumpellin (e) direct recombinant binding assay with GST fused single domains of SNX27 and myc-tagged VPS26, VPS29 and native 
VPS35. (f) GFP-tagged VPS26 and the N-and C-terminal lobe of VPS26 were analyzed for binding to SNX27 (g) endogenous SNX27 and overexpressed mcherry-SNX27 were precipitated with a GFP fused PDZ ligand and analyzed for binding to the indicated proteins. As a control, GFP-tagged SNX27 and SNX27 $\triangle \mathrm{PDZ}$ were precipitated with GFP-trap beads. (h) The GFP -tagged PDZ domains of SNX27 and NHERF1 and NHERF2 were analyzed for binding to VPS26 and VPS35 (i) GFP-tagged SNX27 and SNX27 $67-77$ were analzed for binding to retromer, WASH complex member Strumpellin and PDZ ligand harbouring membrane proteins MRP4 and KIDINS220. All uncropped blots are shown in Fig. S7. 


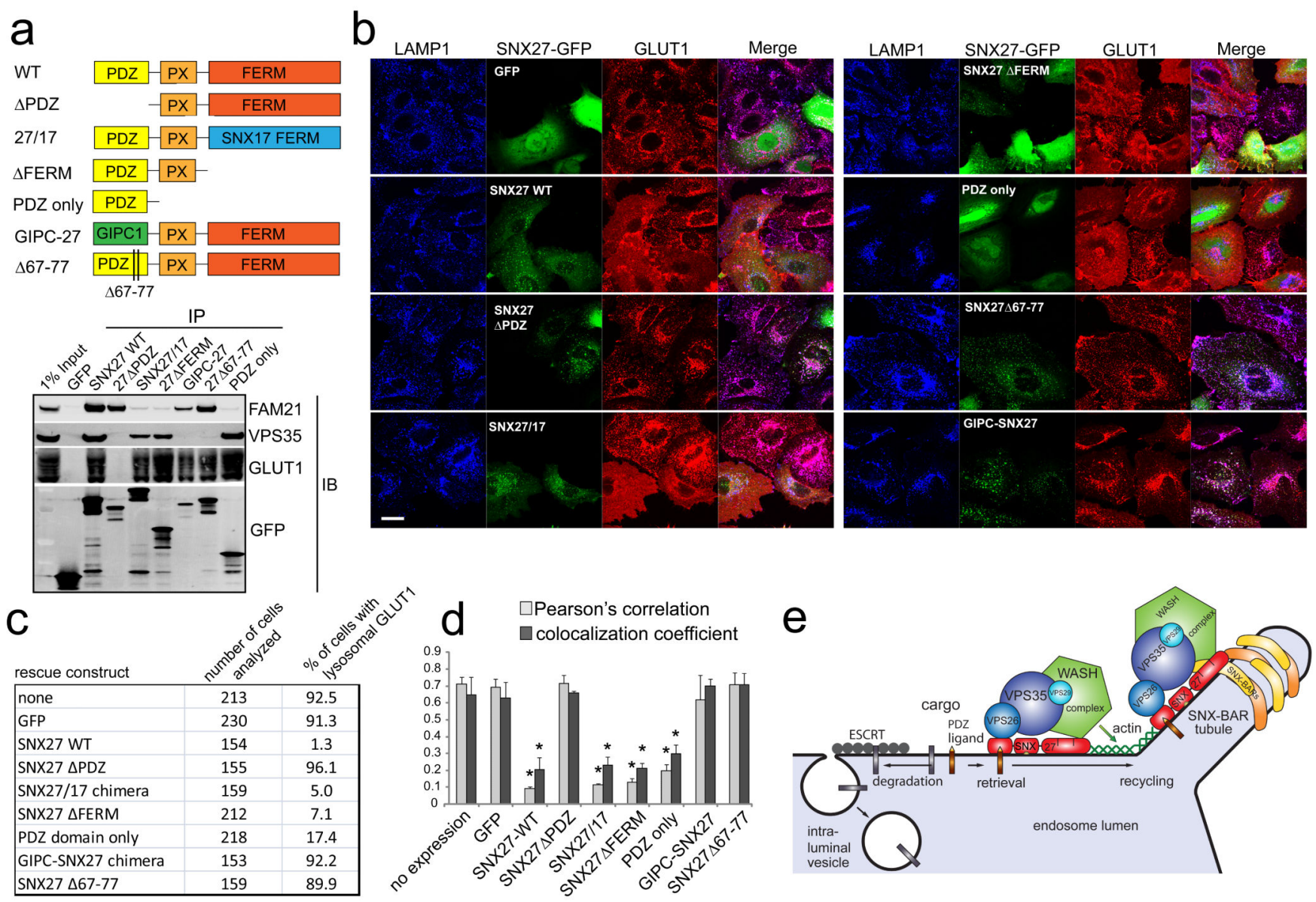

Fig. 8. The interaction of the SNX27 PDZ domain with the VPS26:VPS29:VPS35 complex primary mode of rescuing lysosomal entry of SNX27 cargo

(a) Overview of the GFP-tagged SNX27 constructs used in suppression and rescue experiments (upper panel) and interaction analysis of the indicated SNX27 mutants with Glut1, retromer component VPS35 and WASH component Fam21 (lower panel, uncropped blots are shown in Fig. S8). (b) Rescue experiments based on accumulation of endogenous Glut1 in LAMP1 positive lysosomes in SNX27 deficient cells. SNX27 was suppressed with siRNA and the indicated siRNA resistant GFP-tagged SNX27 constructs were re-expressed. (c) The indicated number of cells for each condition was visually assessed for lysosomal Glut1 accumulation and expression of the respective GFP-tagged rescue constructs. The table shows the results of one representative experiment (d) Colocalization analysis of endogenous Glut1 and LAMP1 in SNX27 depleted cells expressing the indicated rescue constructs. The graph represents the mean of three independent experiments with 20 individual cells each. $(n=3$, * indicates $\mathrm{p}<0.05$, unpaired t-test; error bars $=$ s.d., Scale bar $=$ $10 \mu \mathrm{m}$, individual data points shown in statistics source data). (e) Model of SNX27-retromer based lysosomal retrieval and subsequent transport. 